\title{
Political Extremism in the 1920s and 1930s: Do German Lessons Generalize?
}

\author{
ALAN DE BROMHEAD, BARRY EICHENGREEN, AND \\ KEVIN H. O'ROURKE
}

We examine the impact of the Great Depression on the share of votes for rightwing extremists in elections in the 1920s and 1930s. We confirm the existence of a link between political extremism and economic hard times as captured by growth or contraction of the economy. What mattered was not simply growth at the time of the election, but cumulative growth performance. The impact was greatest in countries with relatively short histories of democracy, with electoral systems that created low hurdles to parliamentary representation, and which had been on the losing side in World War I.

$T_{1}$ he impact of the global credit crisis and Great Recession has been more than just economic. In both parliamentary and presidential democracies, governments have been ousted. Hard economic times have increased political polarization and bred support for nationalist and right-wing political parties. All this gives rise to fears that economic hard times will feed political extremism, as it did in the 1930s.

Memories of the $1930 \mathrm{~s}$ inform much contemporary political commentary, just as they inform recent economic commentary. But exactly what impact the interwar depression and economic crisis had on the electoral fortunes of extremist parties has not been systematically studied. ${ }^{1}$ Many of our intuitions about the links between

The Journal of Economic History, Vol. 73, No. 2 (June 2013). (C) The Economic History Association. doi: 10.1017/S0022050713000302. This is an Open Access article, distributed under the terms of the Creative Commons Attribution license (http://creativecommons.org/ licenses/by/4.0/), which permits unrestricted re-use, distribution, and reproduction in any medium, provided the original work is properly cited.

Alan de Bromhead is Doctor of Philosophy Research Student in Economic and Social History, Mansfield College, University of Oxford, Oxford OX1 3TF, United Kingdom. E-mail: alan.debromhead@mansfield.ox.ac.uk. Barry Eichengreen is Professor of Economics and Political Science, Department of Economics, University of California, Berkeley, 549 Evans Hall 3880, Berkeley, CA 94720-3880. E-mail: eichengr@econ.berkeley.edu. Kevin H. O’Rourke is Professor of Economic History, All Souls College, Oxford OX1 4AL, United Kingdom. E-mail: kevin.orourke@all-souls.ox.ac.uk.

A preliminary version of this article was presented at the conference on "The Ethics of Economics," held in honor of Avner Offer, on October 52011 at All Souls College Oxford. We thank the organizers and the conference participants for helpful comments. We also thank Ken Benoit, Carles Boix, Giovanni Capoccia, Kevin Denny, Rui Esteves, James Fenske, Bill Greene, Doug Irwin, Martin Ivanov, David Leblang, Matthias Morys, Denis Nekipelov, Carol Newman, Avner Offer, Florian Ploeckl, Hans-Joachim Voth, Niko Wolf, the editor, and two anonymous referees, for helpful comments, suggestions, and advice regarding both data and econometrics. The research leading to these results has received funding from the European Research Council under the European Union's Seventh Framework Programme (FP7/20072013) / ERC grant agreement no. 249546. The usual disclaimer applies.

${ }^{1}$ To be sure, there are statistical studies linking unemployment to the rise of the National 
the Depression and political extremism are informed by the case of Germany. There, both communists and fascists saw their vote shares increase sharply as the economic crisis deepened after 1929. The view that this link was causal is widely shared. And the horrific consequences of what followed have led observers, whether consciously or not, to generalize from the German experience.

But was what was true of Germany - that bad economic times fed support for political extremists on both the left and right-also true of other countries? Germany, it can be argued, was distinctive. It was a country in which the Great Depression was unusually severe. A reactionary agrarian aristocracy is said to have hindered the development of democratic culture before 1914. Its population was divided by religion, class, and ideology. The experience of defeat in World War I had a radicalizing effect. The Weimar Republic's electoral system made it easy for small parties to enter Parliament. All these factors have been suggested as reasons for the rise of political extremism in interwar Germany. But it is not clear to what extent one can generalize from German experience and conclude that they were important elsewhere.

Answering such questions is our goal in this article. We study the share of votes for extremists in elections in 28 countries between World Wars I and II, focusing on right- and left-wing anti-system partiesthat is, parties explicitly advocating the overthrow of a country's political system. $^{2}$

Consistent with German experience, we find a link between right-wing political extremism and economic conditions, as captured by the change in GDP. Importantly, however, what mattered for right-wing anti-system party support was not just deterioration in economic conditions lasting a year or two, but economic conditions over the longer run.

In addition, the structure of the voting system shaped the electoral success of right-wing anti-system parties, as the German literature suggests. Specifically, we find that the minimum effective

Socialists in Germany, along with competing studies disputing that link (see, for example, King et al. 2008; O'Loughlin 2000; and Stögbauer 2001). Qualitative studies analyze the breakdown of democracy and the rise of authoritarianism in Germany and elsewhere (see inter alia Linz and Stepan 1978; Berg-Schlosser and Mitchell 2000, 2002; Bermeo 2003). A few analyses have attempted to connect macroeconomic distress to political outcomes more broadly (see e.g., Berg-Schlosser and Mitchell 2000). But a systematic study that looks across countries and asks not just whether there was a link between the interwar business cycle and the rise of extremist parties, but also whether there were economic, political, and social conditions under which that link was especially tight, has not been undertaken to our knowledge. See, however, the discussion below.

${ }^{2}$ In practice, we focus on fascist and other extremist parties on the right and Communist parties on the left, as explained below. 
electoral threshold, defined in terms of the share of the vote that a party had to achieve in order to gain parliamentary representation, mattered for the size of the fascist vote and mattered even more for the extent to which this vote translated into seats.

The effect of the Depression on political outcomes was further shaped by historical circumstances, again as the German literature suggests. It depended on whether a country had long experience with democracy and specifically on whether it had been democratic prior to 1914. It was conditioned by whether the country had been on the losing side in World War I. These factors mattered not just in Germany but more broadly, although they mattered in different countries in different ways.

In contrast, other characteristics of German society, such as religious divisions or the existence of a pre-1914 Junker-style agrarian elite, do not appear to have had a systematic impact on the extremist vote when we look across countries. In addition, the strong effect of the Depression on support for left-wing anti-system parties, emphasized in the literature on Germany, does not appear to have been as evident in other countries. Communist electoral gains after 1929 were not especially impressive, a few prominent exceptions notwithstanding, despite the fact that the Depression was global and understood at the time as representing a crisis of capitalism. The link between the depth of the slump and support for right-wing extremists evident in the data is not equally apparent for left-wing extremists. This emphasis in the German literature does not generalize, in other words. ${ }^{3}$

\section{EXPLANATIONS}

Explanations for political extremism in this period fall into five broad categories: economic factors, social cleavages, external influences, electoral systems, and institutional/cultural inheritances. Authors from S. M. Lipset (1959) to D. Acemoglu and J. A. Robinson (2006) have suggested that the more economically developed (more "modern") a nation, the greater the likelihood that democracy will not only be established but be secured. Attempts to capture modernization in the sense of Lipset have used GDP per capita, education, and urbanization as proxies. Urbanization is also related to the work of B. Moore (1966), who argued that democracy and agrarian society are a difficult marriage owing to the interest of large landowners in maintaining their monopoly of land ownership. According to

\footnotetext{
${ }^{3}$ This fact leads us to focus in most of the article on the determinants of the rise in support for right-wing anti-system parties, although we also note our findings for Communist parties.
} 
Moore, capitalist authoritarianism emerges when conservative and politically powerful large landowners, the military, and a bourgeoisie of medium strength form a coalition. Fascism was the attempt to make such "reaction and conservatism popular and plebeian" (Moore 1966, p. 447). His view is consistent with the Sonderweg approach to German political development according to which a reactionary Junker-dominated elite slowed the development of liberal democracy before World War I. ${ }^{4}$

Support for extremist parties has also been prominently linked to economic performance. The difficult economic conditions of the interwar years are widely cited as a factor in the rise of fascist parties (Frey and Weck 1983; Payne 1996). Arguments connecting unemployment to disaffection with democratic systems are widespread (see, for example, Berg-Schlosser and Mitchell 2000). High inflation is seen as undermining confidence in the ability of mainstream parties to manage the economy in the 1920s. The collapse of prices, production, and financial stability in the 1930s is seen as then working in the same direction. An important determinant of the rise of anti-system parties in the 1930s may thus have been how much economic policy space governments had to counter the slump. K. Borchardt (1991) famously emphasizes German Chancellor Heinrich Brüning's lack of room for maneuver under the gold standard as resulting in deflationary overkill that contributed to the Nazi's rise to power. ${ }^{5}$

A second set of explanations emphasizes social differentiation. Ethno-linguistic, religious, and class cleavages, in this view, are fault lines complicating the development of social consensus and hindering the adoption of a concerted response to economic crisis. ${ }^{6}$ This line of argument features prominently in the literature on post-World War I Europe, where new nations were created with little regard for ethnic and religious considerations. That the resulting populations were heterogeneous posed a challenge for newly established democratic systems. $^{7}$

\footnotetext{
${ }^{4}$ For a critique of the Sonderweg approach, see Kocka (1988). Considerable controversy surrounds the question of whether these arguments can be applied to the interwar years and to just how they must be adapted in order to do so (see inter alia Luebbert 1987).

${ }^{5}$ At the same time, there are dissenters. Bermeo (2003, p. 22) argues that "economic performance failures are not consistently powerful predictors of either democracy's decline or the growth of anti-democratic support among ordinary people."

${ }^{6}$ For a critical examination of the thesis, see Gerrits and Wolffram (2005).

${ }^{7}$ In a recent paper, Voigtlaender and Voth (2011) uncover a striking correlation between anti-Semitic pogroms during the Black Death and votes for the Nazis in the 1920s, suggesting a depressingly efficient cultural transmission mechanism preserving anti-Semitic attitudes over time.
} 
Third, the legacy of the First World War receives considerable attention as a factor shaping the interwar political landscape (Holzer 2002). Warring nations suffered catastrophic losses of men and matériel as well as domestic hardship. Allied blockade subjected the civilian populations of Germany and Austria-Hungary to painful shortages, and rather than ending with the Armistice, these continued until Germany agreed to sign the Treaty of Versailles. Combatants on both sides returned home feeling that their governments had failed to adequately protect them. Since they had been acclimatized by military service to authoritarian forms of organization, when they experienced economic hardships they blamed their governments for failing to provide adequate economic security and entertained authoritarian alternatives (James and Müller-Luckner 2002).

War also had a financial legacy. High levels of indebtedness placed countries on an unstable financial footing and limited the ability of governments to produce favorable economic outcomes. More generally, the terms of the postwar settlement have been seen as contributing to the rise of fascism and political instability (Boemeke 1998). The Versailles Treaty dissolved the Austro-Hungarian Empire and arbitrarily redrew borders, fanning resentment, tension, and instability. Ethnic groups were splintered by new borders, and successor states of the Austro-Hungarian Empire were saddled with high levels of ethnic fractionalization. Reparations and limits on national autonomy were seen as excessively harsh by the defeated powers, assuring political support for nationalist campaigns for annulment. In extreme cases like Germany, the Versailles Treaty was identified with parliamentary democracy, ultimately posing a threat to the Weimar State itself (Holzer 2002).

Fourth, certain political and constitutional systems created more scope for anti-system parties to gain influence. F. A. Hermens (1941) famously held that proportional representation electoral systems led to high levels of party fractionalism and government instability and fostered the rise of anti-system parties. ${ }^{8}$ Lipset (1959) similarly suggested that proportional representation amplified the voice of narrow interests across the political spectrum. ${ }^{9}$

\footnotetext{
${ }^{8}$ Karvonen and Quenter (2002) similarly focus on the impact of electoral and party systems; they too suggest that proportional representation leads to party fragmentation that in turn gives rise to political extremism, although not all their evidence is consistent with the hypothesis.

${ }^{9}$ The steps that incumbent governments took in defense of democracy can also explain the survival of some democratic systems and the failure of others (Capoccia 2005). In some cases, the rise of extremist parties was countered by incumbent governments both through coercion of extremist groups and by the outright banning of political parties. In some cases democratic rights were suspended, it was claimed, in order to safeguard the political system.
} 
Fifth and finally, institutional and cultural inheritances militated in favor of or against extremist parties. An influential tradition associated with G. A. Almond and S. Verba (1989) argues that political culture is an important determinant of the durability of democracies. The "civic culture" which for these authors is a crucial ingredient of democratic stability is transmitted between generations in the home, in schools, and in the broader society, in part as a result of the exposure of people to the democratic system itself (Almond and Verba 1989, pp. 367-68). L. Diamond (1999) also stresses the role of political culture in consolidating democracy, arguing that the embrace of democratic values will be enhanced by exposure to successful democracy. In a similar vein, R. D. Putnam et al. (1993) argue that social capital is essential for making democracy work effectively. More recently, T. Persson and G. Tabellini (2009) have argued that countries with longer histories of democracy accumulate democratic capital, which increases the probability that they will remain democratic. These analyses suggest that anti-system extremists could have benefitted more from the Depression in countries without a well-developed political tradition and poorly endowed with social capital.

\section{PREVIOUS EMPIRICAL ANALYSES}

We are unaware of other multivariate statistical studies of the rise of extremist voting patterns during the interwar period. ${ }^{10} \mathrm{R}$. Jackman and K. Volpert (1996) examine the determinants of the success of extreme right-wing parties between 1970 and 1990. They estimate a Tobit model and find that votes for extreme right-wing parties are negatively related to the electoral threshold and positively related to the unemployment rate, and that the effective number of parties (the degree of multi-partism) is positively associated with a higher extreme-right vote. Acknowledging that electoral thresholds and multi-partism may be interdependent, they also analyze the interaction of the two variables. ${ }^{11}$ They find that while electoral thresholds have little impact on the vote for extreme right-wing parties when the effective number of parties is low, higher thresholds have a significant dampening effect on such votes when the effective number of parties is high. Similarly, while the

\footnotetext{
${ }^{10}$ Berg-Schlosser and Mitchell (2000, 2002) use qualitative-comparative analysis and event histories to analyze factors influencing the breakdown of democratic systems, finding support for modernization theory as well as for the idea that the depth of social cleavages is positively associated with democratic breakdown. However, the focus of our article is on extremist voting rather than on the breakdown of democracy per se.

${ }^{11}$ This observation of potential interdependence draws on Lijphart's (1994) study of post-World War II electoral systems.
} 
effective number of parties does not have much effect on the extreme right-wing vote in the presence of high effective thresholds, it does have such an effect when electoral thresholds are low. ${ }^{12}$

M. Golder (2003) similarly estimates a Tobit model of the vote shares of extreme right-wing parties in Western Europe between 1970 and 2000. He finds that immigration and unemployment are important for explaining their electoral performance. However, his results suggest that unemployment has no effect on right-wing populist votes when the number of immigrants is low: it is the interaction between economic hard times and the presence of immigrants that boosts extremism. P. Knigge (1998), in contrast, rejects the hypothesis that unemployment breeds support for right-wing parties.

J. Ponticelli and H.-J. Voth (2011) look not at electoral outcomes but at indicators of social unrest (demonstrations, assassinations, riots, general strikes, and attempted revolutions) between 1919 and 2009. They relate these to changes in GDP and other variables (including measures of fiscal austerity, which is their particular focus). While their measures of social unrest are not the same as the electoral outcomes of concern to us here, they are likely to be correlated. It is suggestive therefore that in most specifications they find a negative correlation between growth and social unrest even when controlling for other factors.

We conclude that while there are some studies of the link between economic conditions and support for anti-system parties in other periods, a systematic investigation of the connections between the interwar slump and political extremism internationally has yet to be undertaken.

\section{ELECTORAL DATA}

Our data set is made up of 171 elections in 28 countries between 1919 and 1939. The sample is weighted toward Europe, since interwar elections were disproportionately European, but we also include observations for other regions-North America, Latin America, Australia, and New Zealand (all elections for which we could obtain information)wherever possible. The data on election results are compiled from G. Capoccia (2005), T. J. Mackie and R. Rose (1991), D. Nohlen (2005), and Nohlen and P. Stöver (2010), supplemented by D. Sternberger and B. Vogel (1969) for Yugoslavia and the Czech Statistical Office website for Czechoslovakia.

\footnotetext{
${ }^{12}$ For example, under proportional representation.
} 
Anti-system parties are defined by G. Sartori (1976) as parties seeking to change not the government but the system of government. They include fascist and secessionist parties on the right and communist parties on the left. Our particular interest is in extremist right-wing (fascist) anti-system parties, ranging from obvious cases like the NSDAP in Germany to the Arrow Cross in Hungary and the Iron Guard in Romania. ${ }^{13}$ While not all of these extremist right-wing anti-system parties were fascist, strictly speaking, for the sake of brevity we refer interchangeably to "fascists," "extreme right-wing parties," and "right-wing anti-system parties." The main sources used to classify parties are Capoccia $(2002,2005) .{ }^{14}$ Appendix Table 1 lists for each country the parties classified as anti-system on both the left and right.

Tables 1-3 show the changes in seats and votes across the Great Depression divide for all anti-system parties (including secessionists, though these account for a trivial share of the vote); for fascist parties; and for communist parties. In each case, we compare the last election prior to 1929 with the post-1929 election in which the relevant party or parties achieved their peak vote share. ${ }^{15}$ Tables 1 and 3 report means with and without Finland, since the Finnish Communist Party, which had been very large before 1929, was suppressed in $1930 .{ }^{16}$

Table 1 shows that seats and votes for anti-system parties rose significantly following the onset of the Depression in 1929. The means rose from fewer than 4 percent to almost 11 percent, and the medians from less than 2 percent to over 7 percent. The same increases are evident for fascist and communist parties separately (Tables 2 and 3), although the increase across the 1929 watershed is more pronounced for the fascist parties in Table $2 .^{17}$

\footnotetext{
${ }^{13}$ The classification of parties is not always straightforward. An example is Ioannis Metaxas' Free Thinkers' Party in Greece. Although there is some debate as to whether this party should be defined as "fascist" or "ultra-nationalist," its extremist nature justifies its inclusion as a far-right anti-system party in our analysis. As our classification is based on party ideologies which may not have been constant over time, some parties are classified as anti-system in specific periods only. This applies, for example, to the DNVP in Germany between 1929 and 1933, whose ideological shift to the right ultimately facilitated the seizure of power by the NSDAP in 1933. We only consider secessionist parties in our formal statistical analysis if they were also "fascist."

${ }^{14}$ Professor Capoccia was kind enough to provide us with the underlying data. In Sartori's classification, monarchists are also defined as anti-system, but we only consider them in the analysis if they also became fascist.

${ }^{15}$ For Germany, we consider the July and November 1932 elections, depending on the party or parties being considered, since these were more accurate expressions of popular opinion than the 1933 election.

${ }^{16}$ A law of January 1930 gave the government the power to dissolve associations promoting illegal or immoral activity, and that law was used to dissolve all communist groups and associations. A law of November 1930 then declared the communists ineligible for parliament.

${ }^{17}$ Suggesting where we are likely to see the largest effects in the statistical analysis that follows.
} 
TABLE 1

ELECTION RESULTS FOR ALL ANTI-SYSTEM PARTIES

\begin{tabular}{|c|c|c|c|c|c|c|c|c|}
\hline \multirow[b]{2}{*}{ Country } & \multicolumn{3}{|c|}{ Last Pre-1929 } & \multicolumn{3}{|c|}{ Peak Post-1929 } & \multirow{2}{*}{$\begin{array}{l}\text { Change } \\
\text { in Seats }\end{array}$} & \multirow{2}{*}{$\begin{array}{l}\text { Change } \\
\text { in Votes }\end{array}$} \\
\hline & $\%$ Seats & $\%$ Votes & Year & $\%$ Seats & $\%$ Votes & Year & & \\
\hline Argentina & 0 & 0 & 1928 & 0 & 0.5 & 1930 & 0 & 0.5 \\
\hline Australia & 0 & 0 & 1929 & 0 & 1.3 & 1934 & 0 & 1.3 \\
\hline Austria & 7.2 & 8.5 & $*$ & 10.8 & 9.8 & 1930 & 3.6 & 1.4 \\
\hline Belgium & 6.4 & 8.2 & 1929 & 22.8 & 24.7 & 1936 & 16.4 & 16.5 \\
\hline Bulgaria & 0 & 2.5 & 1927 & 11.4 & 13 & 1931 & 11.4 & 10.5 \\
\hline Canada & 0 & 0 & 1926 & 0 & 0.7 & 1935 & 0 & 0.7 \\
\hline Chile & 0 & 0 & 1925 & 8.2 & 7.7 & 1937 & 8.2 & 7.7 \\
\hline Czechoslovakia & 16 & - & 1929 & 32.4 & 25.5 & 1935 & 16.4 & - \\
\hline Denmark & 0 & 0.3 & 1929 & 2.7 & 4.2 & 1939 & 2.7 & 3.9 \\
\hline Finland & 25.5 & 28 & 1929 & 21 & 19 & 1930 & -4.5 & -9 \\
\hline France & 8.9 & - & 1928 & 19.8 & - & 1936 & 10.9 & - \\
\hline Germany & 13.4 & 13.2 & 1928 & 59.6 & 58.3 & 1932 & 46.2 & 45.1 \\
\hline Greece & 0.4 & 6.7 & 1928 & 7.3 & 9.7 & 1936 & 6.9 & 3 \\
\hline Hungary & 0.8 & 3.8 & 1926 & 17.4 & 22.8 & 1939 & 16.6 & 19 \\
\hline Ireland & 0.7 & 1.1 & 1927 & 0 & 0.1 & 1932 & -0.7 & -1 \\
\hline Italy & 11 & 6.2 & 1921 & NA & NA & & NA & NA \\
\hline The Netherlands & 2 & 2 & 1929 & 7 & 7.6 & 1937 & 5 & 5.6 \\
\hline New Zealand & 0 & 0 & 1928 & 0 & 0.1 & 1935 & 0 & 0.1 \\
\hline Norway & 2 & 4 & 1927 & 0 & 4 & 1933 & -2 & 0 \\
\hline Poland & 1.1 & 1.9 & 1928 & NA & NA & & NA & NA \\
\hline Romania & 0 & 1.2 & 1928 & 27.2 & 25.1 & 1937 & 27.2 & 23.9 \\
\hline Spain & - & - & & 16.2 & - & 1936 & - & - \\
\hline Sweden & 3.5 & 6.4 & 1928 & 3.5 & 8.9 & 1932 & 0 & 2.5 \\
\hline Switzerland & 1 & 1.8 & 1928 & 2.1 & 2.6 & 1939 & 1.1 & 0.8 \\
\hline United Kingdom & 0 & 0.2 & 1929 & 0.2 & 0.1 & 1935 & 0.2 & -0.1 \\
\hline United States & 0 & 0 & 1928 & 0 & 0 & & 0 & 0 \\
\hline Uruguay & 0.8 & 1.3 & 1928 & 1.6 & 2 & 1931 & 0.8 & 0.7 \\
\hline Yugoslavia & 0 & 0 & 1927 & NA & NA & & NA & NA \\
\hline $\begin{array}{l}\text { Mean } \\
\text { Mean }\end{array}$ & 3.7 & 3.9 & & 10.8 & 10.8 & & 6.9 & 6.1 \\
\hline (Ex. Finland) & 2.9 & 2.9 & & 10.4 & 10.4 & & 7.4 & 6.8 \\
\hline Median & 0.8 & 1.8 & & 7.0 & 7.6 & & 1.9 & 1.4 \\
\hline
\end{tabular}

Notes: * Last votes data are for 1923, last seats data for 1927. "Pre-1929" elections include elections held in 1929. The results include votes for secessionist parties, not included in either Table 2 or 3. - indicates missing data. NA indicates that no democratic elections were held after 1929. 
TABLE 2

ELECTION RESULTS FOR FASCIST ANTI-SYSTEM PARTIES

\begin{tabular}{|c|c|c|c|c|c|c|c|c|}
\hline \multirow[b]{2}{*}{ Country } & \multicolumn{3}{|c|}{ Last Pre-1929 } & \multicolumn{3}{|c|}{ Peak Post-1929 } & \multirow{2}{*}{$\begin{array}{l}\text { Change } \\
\text { in Seats }\end{array}$} & \multirow{2}{*}{$\begin{array}{l}\text { Change } \\
\text { in Votes }\end{array}$} \\
\hline & $\%$ Seats & $\%$ Votes & Year & $\%$ Seats & $\%$ Votes & Year & & \\
\hline Argentina & 0 & 0 & 1928 & 0 & 0 & 1930 & 0 & 0 \\
\hline Australia & 0 & 0 & 1929 & 0 & 0 & 1934 & 0 & 0 \\
\hline Austria & 0 & 0 & $*$ & 4.8 & 9.2 & 1930 & 4.8 & 9.2 \\
\hline Belgium & 0 & 0 & 1929 & 18.3 & 18.6 & 1936 & 18.3 & 18.6 \\
\hline Bulgaria & 0 & 0 & 1927 & 0 & 0 & 1931 & 0 & 0 \\
\hline Canada & 0 & 0 & 1926 & 0 & 0 & 1935 & 0 & 0 \\
\hline Chile & 0 & 0 & 1925 & 4 & 3.5 & 1937 & 4 & 3.5 \\
\hline Czechoslovakia & 6 & - & 1929 & 22.4 & 15.18 & 1935 & 16.4 & - \\
\hline Denmark & 0 & 0 & 1929 & 0.7 & 1.8 & 1939 & 0.7 & 1.8 \\
\hline Finland & 14 & 14.5 & 1929 & 21 & 18 & 1930 & 7 & 3.5 \\
\hline France & 6.6 & - & 1928 & 7.9 & - & 1936 & 1.3 & - \\
\hline Germany & 2.4 & 2.6 & 1928 & 43.9 & 43.2 & 1932 & 41.5 & 40.6 \\
\hline Greece & 0.4 & 5.3 & 1928 & 2.3 & 3.9 & 1936 & 1.9 & -1.4 \\
\hline Hungary & 0.8 & 3.8 & 1926 & 17.4 & 22.8 & 1939 & 16.6 & 19 \\
\hline Ireland & 0 & 0 & 1927 & 0 & 0 & 1932 & 0 & 0 \\
\hline Italy & 8.2 & 1.6 & 1921 & NA & NA & & NA & NA \\
\hline The Netherlands & 0 & 0 & 1929 & 4 & 4.2 & 1937 & 4 & 4.2 \\
\hline New Zealand & 0 & 0 & 1928 & 0 & 0 & 1935 & 0 & 0 \\
\hline Norway & 0 & 0 & 1927 & 0 & 2.2 & 1933 & 0 & 2.2 \\
\hline Poland & 0 & 0 & 1928 & NA & NA & & NA & NA \\
\hline Romania & 0 & 1.2 & 1928 & 27.2 & 25.1 & 1937 & 27.2 & 23.9 \\
\hline Spain & - & - & & 3.5 & - & 1933 & - & - \\
\hline Sweden & 0 & 0 & 1928 & 0 & 0.7 & 1936 & 0 & 0.7 \\
\hline Switzerland & 0 & 0 & 1928 & 0.5 & 1.5 & 1935 & 0.5 & 1.5 \\
\hline United Kingdom & 0 & 0 & 1929 & 0 & 0 & 1935 & 0 & 0 \\
\hline United States & 0 & 0 & 1928 & 0 & 0 & & 0 & 0 \\
\hline Uruguay & 0 & 0 & 1928 & 0 & 0 & 1931 & 0 & 0 \\
\hline Yugoslavia & 0 & 0 & 1927 & NA & NA & & NA & NA \\
\hline Mean & 1.4 & 1.2 & & 7.1 & 7.4 & & 6.0 & 5.8 \\
\hline Median & 0.0 & 0.0 & & 0.7 & 1.8 & & 0.6 & 1.1 \\
\hline
\end{tabular}

Notes: * Last votes data are for 1923, last seats data for 1927. "Pre-1929" elections include elections held in 1929. - indicates missing data. NA indicates that no democratic elections were held after 1929. 
A considerable variety of country experience is summarized in the tables. In countries like Germany and Czechoslovakia, a relatively high pre-Depression anti-system vote rose even higher after 1929. In Germany, roughly 13 percent of votes and seats went to anti-system parties in 1928, while anti-system parties took almost 60 percent of all votes and seats in November 1932, a huge increase. ${ }^{18}$ In Bulgaria and Romania, anti-system parties that had garnered almost no votes and seats before the Depression won large numbers of votes in its wake. In Ireland and Norway, in contrast, small anti-system parties remained small.

Although fascist and other right-wing parties were often most successful in harnessing anti-system support, this was not uniformly the case. While the extreme right saw its support rise dramatically in Romania, the Communist Party benefited the most from the Depression in neighboring Bulgaria. Although Austria, Belgium, Czechoslovakia, Germany and Hungary all saw substantial increases in the extreme right vote, it was Communists that gained support in Chile, Greece, and France.

\section{EXPLANATORY VARIABLES}

Our aim is to estimate the determinants of vote shares in all interwar elections, not just those in Tables 1 through 3. The explanatory variable of special interest is the change in real GDP. ${ }^{19}$ In addition, we control for the impact of a series of variables corresponding to the five explanations for interwar extremism enumerated above. Lipset (1959, p. 80), in an influential article, writes that the key factors which for him make up economic development and help to sustain democracy, "the factors of industrialization, urbanization, wealth, and education, are so closely interrelated as to form one common factor." Following in this tradition, we gathered data on the percentage of the population that was urbanized. $^{20}$

\footnotetext{
${ }^{18}$ Tables 1-3 rely on Capoccia's classification, according to which the DNVP was not anti-system in 1928 but had become so by 1932 (see above). This switch obviously increases the measured electoral gains of anti-system parties. Alternatively, one could classify not only the DNVP but the DVP as well as anti-system throughout. In this case, the increase in the total anti-system vote in Germany was from 36.1 percent in 1928 to 60.2 percent in November 1932, a still sizeable 24 percentage point increase; while the increase in the anti-system extreme rightwing vote was from 25.5 percent to 44.4 percent in July 1932, a 19 percentage point increase.

${ }^{19}$ GDP data are from Maddison (2010) supplemented by Ivanov (forthcoming) for Bulgaria.

${ }^{20}$ A variable provided in Banks (2011) for the countries in our sample.
} 
TABLE 3

ELECTION RESULTS FOR COMMUNIST PARTIES

\begin{tabular}{|c|c|c|c|c|c|c|c|c|}
\hline \multirow[b]{2}{*}{ Country } & \multicolumn{3}{|c|}{ Last Pre-1929 } & \multicolumn{3}{|c|}{ Peak Post-1929 } & \multirow{2}{*}{$\begin{array}{l}\text { Change } \\
\text { in Seats }\end{array}$} & \multirow{2}{*}{$\begin{array}{l}\text { Change } \\
\text { in Votes }\end{array}$} \\
\hline & $\%$ Seats & $\%$ Votes & Year & $\%$ Seats & $\%$ Votes & Year & & \\
\hline Argentina & 0 & 0 & 1928 & 0 & 0.5 & 1930 & 0 & 0.5 \\
\hline Australia & 0 & 0 & 1929 & 0 & 1.3 & 1934 & 0 & 1.3 \\
\hline Austria & 0 & 0.7 & $*$ & 0 & 0.6 & 1930 & 0 & -0.1 \\
\hline Belgium & 0.5 & 1.9 & 1929 & 4.5 & 6.1 & 1936 & 4 & 4.2 \\
\hline Bulgaria & 0 & 2.5 & 1927 & 11.4 & 13 & 1931 & 11.4 & 10.5 \\
\hline Canada & 0 & 0 & 1926 & 0 & 0.7 & 1935 & 0 & 0.7 \\
\hline Chile & 0 & 0 & 1925 & 4.2 & 4.2 & 1937 & 4.2 & 4.2 \\
\hline Czechoslovakia & 10 & 10.2 & 1929 & 10 & 10.32 & 1935 & 0 & 0.12 \\
\hline Denmark & 0 & 0.3 & 1929 & 2 & 2.4 & 1939 & 2 & 2.1 \\
\hline Finland & 11.5 & 13.5 & 1929 & 0 & 1 & 1930 & -11.5 & -12.5 \\
\hline France & 2.3 & 11.3 & 1928 & 11.8 & 15.3 & 1936 & 9.5 & 4 \\
\hline Germany & 11 & 10.6 & 1928 & 17.1 & 16.9 & 1932 & 6.1 & 6.3 \\
\hline Greece & 0 & 1.4 & 1928 & 5 & 5.8 & 1936 & 5 & 4.4 \\
\hline Hungary & 0 & 0 & 1926 & 0 & 0 & 1939 & 0 & 0 \\
\hline Ireland & 0.7 & 1.1 & 1927 & 0 & 0.1 & 1932 & -0.7 & -1 \\
\hline Italy & 2.8 & 4.6 & 1921 & NA & NA & & NA & NA \\
\hline The Netherlands & 2 & 2 & 1929 & 3 & 3.4 & 1937 & 1 & 1.4 \\
\hline New Zealand & 0 & 0 & 1928 & 0 & 0.1 & 1935 & 0 & 0.1 \\
\hline Norway & 2 & 4 & 1927 & 0 & 1.8 & 1933 & -2 & -2.2 \\
\hline Poland & 1.1 & 1.9 & 1928 & NA & NA & & NA & NA \\
\hline Romania & 0 & 0 & 1928 & 0 & 0 & 1937 & 0 & 0 \\
\hline Spain & - & - & & 3.8 & - & 1936 & - & - \\
\hline Sweden & 3.5 & 6.4 & 1928 & 3.5 & 8.3 & 1932 & 0 & 1.9 \\
\hline Switzerland & 1 & 1.8 & 1928 & 2.1 & 2.6 & 1939 & 1.1 & 0.8 \\
\hline United Kingdom & 0 & 0.2 & 1929 & 0.2 & 0.1 & 1935 & 0.2 & -0.1 \\
\hline United States & 0 & 0 & 1928 & 0 & 0 & & 0 & 0 \\
\hline Uruguay & 0.8 & 1.3 & 1928 & 1.6 & 2 & 1931 & 0.8 & 0.7 \\
\hline Yugoslavia & 0 & 0 & 1927 & NA & NA & & NA & NA \\
\hline $\begin{array}{l}\text { Mean } \\
\text { Mean }\end{array}$ & 1.8 & 2.8 & & 3.2 & 4.0 & & 1.3 & 1.1 \\
\hline (Ex. Finland) & 1.5 & 2.4 & & 3.3 & 4.2 & & 1.9 & 1.7 \\
\hline Median & 0.0 & 1.3 & & 1.6 & 1.9 & & 0.0 & 0.7 \\
\hline
\end{tabular}

Notes: * Last votes data are for 1923, last seats data for 1927. "Pre-1929" elections include elections held in 1929. - indicates missing data. NA indicates that no democratic elections were held after 1929. 
Next, in order to assess Moore's arguments, we classified countries on the basis of whether or not they had an agrarian elite prior to 1914 . We also created dummy variables indicating whether or not countries suffered from religious or ethno-linguistic fractionalization; and whether or not they had been on the losing side in World War I, and had their borders redrawn as a result.

Finally, in order to assess the impact of electoral systems and political histories, we collected data on the effective electoral threshold (the minimum share of the vote a party had to attract in order to gain parliamentary representation), on whether countries had a proportional representation electoral system, and on how long they had been democracies. Data on effective electoral thresholds are from C. Boix (1999), supplemented with our own calculations based on Colomer (2004) and Lijphart (1994). Dummy variables relating to World War I are constructed using individual country histories. Our measures of ethno-linguistic and religious cleavages, whether a country was a democracy before 1914, and the existence of a prewar agrarian elite are based on classifications in D. Berg-Schlosser and J. Mitchell (2002).

\section{VOTING PATTERNS AND COUNTRY CHARACTERISTICS}

Before proceeding to regression analysis, in Table 4 we explore the relationship between country characteristics and the average fascist and communist vote. Since our major interest is in the impact of the Depression on extremist votes, we also look at how these votes changed after 1929 and whether these changing vote shares were related to country characteristics. The table thus presents the results of a series of simple descriptive regressions, in which voting shares are regressed on a post-1929 dummy, country characteristics (one per column), and the interaction between these two variables. Finland is excluded from regressions describing the share of the Communist vote, for reasons mentioned above, while all results are presented for country samples both including and excluding Germany. ${ }^{21}$

\footnotetext{
${ }^{21}$ Sharp-eyed readers will note that in several cases the coefficient on the post-1929 dummy variable is the same no matter whether Germany is included or excluded. This coefficient measures the average increase in the vote share in question after 1929, in countries for which the value of the country characteristic in question is zero. In most cases, the value of the country characteristic for Germany is equal to one, meaning that the inclusion or exclusion of German elections has no effect on this coefficient.
} 
TABLE 4 DETERMINANTS OF ANTI-SYSTEM PARTY VOTE SHARE, 1919-1939

\begin{tabular}{|c|c|c|c|c|c|c|c|c|}
\hline & (1) & (2) & (3) & (4) & (5) & (6) & (7) & (8) \\
\hline Variables & $\begin{array}{c}\text { Prewar } \\
\text { Agricultural } \\
\text { Elite }\end{array}$ & $\begin{array}{c}\text { Above } \\
\text { Median } \\
\text { Urban } \\
\text { Share }\end{array}$ & $\begin{array}{l}\text { Religious } \\
\text { Divide }\end{array}$ & $\begin{array}{l}\text { Ethno- } \\
\text { Linguistic } \\
\text { Divide }\end{array}$ & $\begin{array}{l}\text { WWI } \\
\text { Loser }\end{array}$ & $\begin{array}{c}\text { WWI } \\
\text { Boundary } \\
\text { Changes }\end{array}$ & $\begin{array}{c}\text { PR } \\
\text { Electoral } \\
\text { System }\end{array}$ & $\begin{array}{c}\text { Prewar } \\
\text { Democracy }\end{array}$ \\
\hline \multicolumn{9}{|c|}{ Panel A. Votes for extreme right-wing anti-system parties } \\
\hline $\begin{array}{l}\text { Country } \\
\text { characteristic }\end{array}$ & $\begin{array}{c}0.208 \\
(0.536)\end{array}$ & $\begin{array}{l}-0.383 \\
(0.522)\end{array}$ & $\begin{array}{l}-0.393 \\
(0.522)\end{array}$ & $\begin{array}{l}-0.626 \\
(0.472)\end{array}$ & $\begin{array}{c}0.544 \\
(0.646)\end{array}$ & $\begin{array}{l}1.012 * \\
(0.494)\end{array}$ & $\begin{array}{l}-0.733 \\
(0.752)\end{array}$ & $\begin{array}{c}-1.078 * * \\
(0.523)\end{array}$ \\
\hline Post-1929 & $\begin{array}{l}2.717 \\
(1.668)\end{array}$ & $\begin{array}{l}3.973 * \\
(2.048)\end{array}$ & $\begin{array}{l}4.131 * * \\
(1.609)\end{array}$ & $\begin{array}{l}5.698 \\
(3.405)\end{array}$ & $\begin{array}{l}2.512 * \\
(1.277)\end{array}$ & $\begin{array}{c}2.251 \\
(1.655)\end{array}$ & $\begin{array}{c}1.781 \\
(1.599)\end{array}$ & $\begin{array}{l}10.58 * * \\
(4.718)\end{array}$ \\
\hline $\begin{array}{l}\text { Country } \\
\text { characteristic } \\
\text { *post-1929 }\end{array}$ & $\begin{array}{c}5.845 \\
(5.134)\end{array}$ & $\begin{array}{c}2.160 \\
(4.272)\end{array}$ & $\begin{array}{r}2.582 \\
(5.725)\end{array}$ & $\begin{array}{l}-1.711 \\
(3.893)\end{array}$ & $\begin{array}{l}17.78 * \\
(8.796)\end{array}$ & $\begin{array}{c}6.179 \\
(4.666)\end{array}$ & $\begin{array}{c}5.543 \\
(3.944)\end{array}$ & $\begin{array}{l}-9.184 * \\
(4.801)\end{array}$ \\
\hline Constant & $\begin{array}{c}0.440 \\
(0.452)\end{array}$ & $\begin{array}{r}0.706 \\
(0.437)\end{array}$ & $\begin{array}{r}0.706 \\
(0.429)\end{array}$ & $\begin{array}{l}0.791 * \\
(0.447)\end{array}$ & $\begin{array}{c}0.439 \\
(0.305)\end{array}$ & $\begin{array}{c}-0 \\
(6.30 \mathrm{e}-08)\end{array}$ & $\begin{array}{c}1.033 \\
(0.722)\end{array}$ & $\begin{array}{l}1.078 * * \\
(0.523)\end{array}$ \\
\hline Observations & 159 & 159 & 159 & 159 & 159 & 159 & 159 & 159 \\
\hline$\underline{R \text {-squared }}$ & 0.175 & 0.117 & 0.119 & 0.121 & 0.420 & 0.207 & 0.152 & 0.299 \\
\hline \multicolumn{9}{|c|}{ Panel B. Votes for extreme right-wing anti-system parties excluding Germany } \\
\hline $\begin{array}{l}\text { Country } \\
\text { characteristic }\end{array}$ & $\begin{array}{l}-0.0216 \\
(0.497)\end{array}$ & $\begin{array}{l}-0.686 \\
(0.438)\end{array}$ & $\begin{array}{l}-0.706 \\
(0.430)\end{array}$ & $\begin{array}{l}-0.461 \\
(0.477)\end{array}$ & $\begin{array}{l}-0.0637 \\
(0.420)\end{array}$ & $\begin{array}{c}0.850 \\
(0.514)\end{array}$ & $\begin{array}{l}-0.921 \\
(0.731)\end{array}$ & $\begin{array}{l}-0.912 \\
(0.550)\end{array}$ \\
\hline Post-1929 & $\begin{array}{l}2.717 \\
(1.671)\end{array}$ & $\begin{array}{l}3.973 * \\
(2.051)\end{array}$ & $\begin{array}{l}4.131 * * \\
(1.612)\end{array}$ & $\begin{array}{l}2.466 \\
(1.545)\end{array}$ & $\begin{array}{l}2.512^{*} \\
(1.279)\end{array}$ & $\begin{array}{c}2.251 \\
(1.657)\end{array}$ & $\begin{array}{c}1.781 \\
(1.601)\end{array}$ & $\begin{array}{l}5.949 * * \\
(2.241)\end{array}$ \\
\hline $\begin{array}{l}\text { Country } \\
\text { characteristic } \\
\text { *post-1929 }\end{array}$ & $\begin{array}{r}0.869 \\
(2.330)\end{array}$ & $\begin{array}{l}-1.537 \\
(2.441)\end{array}$ & $\begin{array}{l}-3.182 * \\
(1.777)\end{array}$ & $\begin{array}{c}1.520 \\
(2.441)\end{array}$ & $\begin{array}{l}5.609 * * \\
(2.163)\end{array}$ & $\begin{array}{c}1.912 \\
(2.382)\end{array}$ & $\begin{array}{c}2.083 \\
(2.312)\end{array}$ & $\begin{array}{l}-4.552 * \\
(2.413)\end{array}$ \\
\hline Constant & $\begin{array}{c}0.440 \\
(0.453)\end{array}$ & $\begin{array}{c}0.706 \\
(0.438)\end{array}$ & $\begin{array}{c}0.706 \\
(0.430)\end{array}$ & $\begin{array}{c}0.626 \\
(0.452)\end{array}$ & $\begin{array}{l}0.439 \\
(0.305)\end{array}$ & $\begin{array}{c}-0 \\
(2.23 \mathrm{e}-08)\end{array}$ & $\begin{array}{c}1.033 \\
(0.723)\end{array}$ & $\begin{array}{c}0.912 \\
(0.550)\end{array}$ \\
\hline Observations & 150 & 150 & 150 & 150 & 150 & 150 & 150 & 150 \\
\hline$R$-squared & 0.116 & 0.141 & 0.188 & 0.119 & 0.168 & 0.157 & 0.125 & 0.265 \\
\hline
\end{tabular}

The coefficients can be interpreted as differences between group means. The constant term in column 1 in Panel A, for example, indicates that the average fascist vote share was 0.44 percent before 1929 in countries without a prewar agricultural elite. 
TABLE $4-$ continued

\begin{tabular}{|c|c|c|c|c|c|c|c|c|}
\hline & (1) & (2) & (3) & (4) & (5) & (6) & (7) & (8) \\
\hline Variables & $\begin{array}{c}\text { Prewar } \\
\text { Agricultural } \\
\text { Elite }\end{array}$ & $\begin{array}{c}\text { Above } \\
\text { Median } \\
\text { Urban } \\
\text { Share }\end{array}$ & $\begin{array}{l}\text { Religious } \\
\text { Divide }\end{array}$ & $\begin{array}{l}\text { Ethno- } \\
\text { Linguistic } \\
\text { Divide }\end{array}$ & $\begin{array}{l}\text { WWI } \\
\text { Loser }\end{array}$ & $\begin{array}{c}\text { WWI } \\
\text { Boundary } \\
\text { Changes }\end{array}$ & $\begin{array}{c}\text { PR } \\
\text { Electoral } \\
\text { System }\end{array}$ & $\begin{array}{c}\text { Prewar } \\
\text { Democracy }\end{array}$ \\
\hline \multicolumn{9}{|c|}{ Panel C. Votes for Communist parties excluding Finland } \\
\hline $\begin{array}{l}\text { Country } \\
\text { characteristic }\end{array}$ & $\begin{array}{l}-1.646 \\
(1.466)\end{array}$ & $\begin{array}{l}-1.905 \\
(1.408)\end{array}$ & $\begin{array}{c}2.176 \\
(1.614)\end{array}$ & $\begin{array}{c}0.765 \\
(1.733)\end{array}$ & $\begin{array}{c}4.863^{*} \\
(2.468)\end{array}$ & $\begin{array}{c}2.190 \\
(1.300)\end{array}$ & $\begin{array}{r}2.198 * \\
(1.245)\end{array}$ & $\begin{array}{c}-2.257 \\
(1.529)\end{array}$ \\
\hline Post-1929 & $\begin{array}{l}0.0192 \\
(0.967)\end{array}$ & $\begin{array}{c}0.226 \\
(0.927)\end{array}$ & $\begin{array}{l}1.152^{* *} \\
(0.552)\end{array}$ & $\begin{array}{l}1.587 * * \\
(0.768)\end{array}$ & $\begin{array}{l}0.794 * \\
(0.437)\end{array}$ & $\begin{array}{c}0.409 \\
(0.297)\end{array}$ & $\begin{array}{c}0.907 \\
(0.560)\end{array}$ & $\begin{array}{c}1.324 \\
(1.555)\end{array}$ \\
\hline $\begin{array}{l}\text { Country } \\
\text { characteristic } \\
\text { *post-1929 }\end{array}$ & $\begin{array}{c}1.683 \\
(1.476)\end{array}$ & $\begin{array}{c}1.543 \\
(1.185)\end{array}$ & $\begin{array}{l}-0.648 \\
(1.559)\end{array}$ & $\begin{array}{l}-1.928 \\
(1.361)\end{array}$ & $\begin{array}{c}1.362 \\
(2.800)\end{array}$ & $\begin{array}{c}0.908 \\
(1.182)\end{array}$ & $\begin{array}{c}0.265 \\
(1.244)\end{array}$ & $\begin{array}{c}-0.470 \\
(1.604)\end{array}$ \\
\hline Constant & $\begin{array}{l}3.051^{* *} \\
(1.253)\end{array}$ & $\begin{array}{l}3.075^{* *} \\
(1.165)\end{array}$ & $\begin{array}{l}1.310^{* *} \\
(0.492)\end{array}$ & $\begin{array}{c}1.922 * * * \\
(0.680)\end{array}$ & $\begin{array}{l}1.256^{* * *} \\
(0.403)\end{array}$ & $\begin{array}{l}0.987 * * \\
(0.418)\end{array}$ & $\begin{array}{c}0.836 \\
(0.657)\end{array}$ & $\begin{array}{c}3.457^{* *} \\
(1.439)\end{array}$ \\
\hline Observations & 156 & 156 & 156 & 156 & 156 & 156 & 156 & 156 \\
\hline$R$-squared & 0.029 & 0.036 & 0.054 & 0.020 & 0.232 & 0.093 & 0.073 & 0.083 \\
\hline
\end{tabular}

Panel D. Votes for Communist parties excluding Finland and Germany

\begin{tabular}{lcccccccc}
\hline $\begin{array}{l}\text { Country } \\
\text { characteristic }\end{array}$ & $-2.328^{*}$ & $-2.718^{* *}$ & 1.664 & 1.269 & 4.578 & 1.790 & 1.830 & -1.784 \\
& $(1.312)$ & $(1.184)$ & $(1.756)$ & $(1.675)$ & $(3.398)$ & $(1.359)$ & $(1.275)$ & $(1.639)$ \\
Post-1929 & 0.0192 & 0.226 & $1.152^{* *}$ & $0.963^{*}$ & $0.794^{*}$ & 0.409 & 0.907 & -0.00254 \\
& $(0.968)$ & $(0.929)$ & $(0.552)$ & $(0.512)$ & $(0.438)$ & $(0.297)$ & $(0.561)$ & $(1.214)$ \\
$\begin{array}{l}\text { Country } \\
\quad \text { characteristic }\end{array}$ & & & & & & & & \\
$\quad$ *post-1929 & 0.586 & 1.065 & -1.916 & -1.304 & -1.753 & 0.0923 & -0.619 & 0.856 \\
& $(1.086)$ & $(1.099)$ & $(1.233)$ & $(1.235)$ & $(2.150)$ & $(1.037)$ & $(0.998)$ & $(1.276)$ \\
Constant & $3.051^{* *}$ & $3.075^{* *}$ & $1.310^{* *}$ & $1.418^{* *}$ & $1.256^{* * *}$ & $0.987 * *$ & 0.836 & $2.984 *$ \\
& $(1.255)$ & $(1.167)$ & $(0.493)$ & $(0.508)$ & $(0.403)$ & $(0.419)$ & $(0.658)$ & $(1.555)$ \\
Observations & 147 & 147 & 147 & 147 & 147 & 147 & 147 & 147 \\
$R$-squared & 0.072 & 0.086 & 0.027 & 0.017 & 0.124 & 0.055 & 0.042 & 0.036 \\
\hline$* p<0.1 * * p<0.05 * * * p<0.01$ & & & & & &
\end{tabular}

Note: Robust standard errors clustered by country in parentheses.

Source: See the text.

It was $(0.44+0.208)=0.648$ percent before 1929 in countries with such an elite; it was $(0.44+2.717)=3.157$ percent in countries without such an elite after 1929; and it was $(0.44+0.208+2.717+5.845)=$ 9.21 percent after 1929 in countries with a pre-1914 agrarian elite. 
These are large differences, but the table indicates that they are not statistically significant. More importantly, column 1 in Panel $B$ shows that the difference between countries with and without a pre-1914 agricultural elite vanishes if Germany is excluded from the sample.

As already mentioned, the country characteristics chosen correspond to the five broad explanations for interwar political extremism surveyed earlier. Columns 1 and 2 look at the impact of having had a pre-1914 agrarian elite, and being highly urbanized, variables corresponding to those accounts stressing modernization and the German Sonderweg. Columns 3 and 4 look at the impact of religious and ethno-linguistic fractionalization. Columns 5 and 6 explore the effects of the First World War, looking at whether countries had been on the losing side in the war, and had experienced boundary changes as a result. Column 7 looks at the impact of having a PR electoral system. Finally, column 8 addresses the argument that a history of democracy matters, by including a variable indicating whether or not a country had been a democracy before 1914 .

Panels A and B look at votes for right-wing extremist parties. In all regressions, the post-1929 dummy variable is positive, and in half the cases, it is statistically significant: as we saw in Table 2, the Depression was good for fascists.

But how do the other arguments outlined above fare? There is no support in these results for the arguments, associated with Lipset or Moore, that modernity, as measured by urbanization, or the absence of a pre-1914 agricultural elite, lowered support for fascists. Neither country characteristic has a big effect on its own on the fascist vote share, nor do they have a statistically significant effect after 1929 , nor do they have a big effect after 1929 once Germany is excluded from the sample. ${ }^{22}$ Arguments stressing religious and ethno-linguistic divisions fare just as badly in the context of our 28 -country sample. ${ }^{23}$

In contrast, World War I emerges as having had a major impact on the fascist vote. Before 1929 there was only a trivial difference between the fascist vote in victors and losers, but after 1929 a major gap opened up between the two groups of countries. The fascist vote increased by 2.5 percentage points in countries that had been neutral or on the winning side in World War I, but it rose by more than 8

\footnotetext{
${ }^{22}$ The coefficients in column 2 of Panel B imply that excluding Germany, the fascist vote share after 1929 was slightly more than 2 percentage points lower in more urbanized countries, but as mentioned in the text the effect is both small and statistically insignificant.

${ }^{23}$ Indeed the interaction effect between the post-1929 and religious divide dummies actually becomes negative and statistically significant once Germany is excluded from the sample (column 3, Panel B).
} 
percentage points in countries that had been on the losing side, even when Germany is excluded from the sample. ${ }^{24}$

Once Germany is excluded from the sample, there is not much support for the argument that proportional representation helped fascists. In contrast, there is substantial support for the view that a history of democracy helped insulate countries from extremist pressures, and that it was younger democracies that were particularly exposed to the risk of right-wing extremism. Votes for fascists increased much less after 1929 in countries with a history of prewar democracy. Indeed, comparing the sizes of the positive coefficient on the post-1929 dummy variable, and the negative coefficient on the interaction effect, suggests that there was only a very small increase in the fascist vote after 1929 (less than 1.5 percentage points) in those countries that had been democratic before 1914.

Panels C and D consider the communist vote. The contrast with the previous results is striking. The coefficients on the post-1929 dummy are much smaller, and in one case even negative. None of the interaction terms is statistically significant, and all are quite small. Evidently, the Depression was of much less help to communist than fascist parties. On the other hand, the communist vote was higher in countries that had been on the losing side in World War I, just as was true for fascists: extremists of all stripes benefited from that calamity. The difference is that while this war effect only showed up after 1929 in the case of fascists, it was present before that date in the case of Communists. Indeed, once Germany is excluded the war bonus enjoyed by Communists became smaller after 1929, in sharp contrast with the fascist experience. Communist parties also enjoyed a higher share of the vote in countries with PR electoral systems. ${ }^{25}$

Thus, while the Depression clearly benefitted extreme right-wing parties, it favored Communist parties less. The two country characteristics that emerge as being most important in determining extremist support were political traditions and recent military history. Fascists enjoyed higher vote shares in countries without a prewar democratic tradition, especially after 1929. This evidence is consistent with the arguments of Almond and Verba (1989), Persson and Tabellini (2009), and others

\footnotetext{
${ }^{24}$ Column 5, Panel B. While the fascist vote was higher both before and after 1929 in countries which had experienced boundary changes following the war, this effect is not robust to dropping Germany from the sample.

${ }^{25}$ Excluding Germany only changes the coefficients on having lost the war, and having a PR electoral system, a little, but the coefficients become statistically insignificant, with $p$-values of 0.190 and 0.164 respectively. On the other hand, excluding Germany, the communist vote share was statistically significantly lower in countries with a prewar agricultural elite, and in more urbanized countries.
} 
emphasizing the influence of political culture and democratic capital on the durability of democracy.

The legacy of the war also comes through clearly: countries on the losing side in 1918 had higher Communist votes throughout and saw a higher increase in the fascist vote after 1929. As Stefano Bartolini puts it $(2000$, p. 90), the "patriotic slaughter" of the war radicalized opinion, but "the extent of this alienation depended to a large extent on the war position and involvement of each country, that is, whether it was belligerent or neutral, defeated or victorious...The "revolutionary accumulation" was likely to be stronger among countries at war than among neutral countries and stronger among defeated than among victorious ones." Just as any satisfactory account of the Great Depression has to begin with the economic, social, and political impacts of the Great War, so does any account of interwar extremism have to begin with the legacies of that catastrophe.

\section{REGRESSION RESULTS}

Thus far we have explored average changes in voting behavior after 1929, a watershed year for the world economy. But to firmly establish the relationship between economic hard times and extremist voting, we need to go further. Some countries experienced more severe depressions than others, while different countries entered the Depression and began recovering at different dates. Simply comparing pre- and post-1929 experience does not allow us to account for this. Since other forces at work after 1929 could have contributed to the fascist vote, it is important to see if there is still a role for the business cycle in determining this vote after having controlled for such factors.

Because the vote share of anti-system parties could be zero, rendering ordinary least squares or a logit transformation of vote shares inappropriate, we follow Jackman and Volpert (1996) and Golder (2003) in using a Tobit model. ${ }^{26}$ In particular, we use the semi-parametric fixed-effects Tobit estimator proposed by B. Honoré (1992). ${ }^{27}$ This may be asking a lot of our data, given that in a typical regression we have between 125 and 150 observations and around 25 countries. But there were surely unobserved country-specific factors which affected voting patterns, justifying the method. Owing to our fixed-effects approach, we exclude time-invariant variables from the regressions, although we can interact those time-invariant variables with changes in GDP.

\footnotetext{
${ }^{26}$ As Wooldridge (2010, chapter 17) emphasizes, Tobit models are appropriate not just when the data are censored, but when there are corner solutions.

${ }^{27}$ The Honoré estimator is implemented using the pantob command in Stata.
} 
The dependent variable in Table 5 is the share of the vote going to fascist anti-system parties. ${ }^{28}$ Successive columns explore the relationship between this variable and GDP growth in the previous one (columns 1-3), two (columns 4-6), and three (columns 7-9) years using the semi-parametric fixed effects Tobit estimator. The control variables, which are successively added, are urbanization and the effective electoral threshold. Although Honoré's method does not permit us to use annual dummies, we also want to take account of the possibility that the early and late 1920s were different from the early and late 1930 s for reasons other than the deteriorating economy. We therefore divide our time period into four segments: 1919-1924, 1925-1928, 1929-1933, and 1934-1939, representing the years of postwar turmoil and hyperinflation; the period of recovery and reconstruction of the international economy; the Great Depression; and the gradual recovery from the 1933 trough, respectively. We construct dummy variables for each of these four periods with the last as the omitted alternative.

Consistent with Table 4, the results lend little support to the hypothesis that urbanization (as a proxy for modernization, and here entered into the regression as a continuous variable) reduced votes for right-wing anti-system parties. The variable has the wrong sign in columns 2, 5, and 8 and is statistically significant in two cases, while in columns 3, 6, and 9 it has the right sign but is small and insignificant. A possible objection to this negative finding is that by including fixed effects, we are stripping out differences in average levels of urbanization across countries and relying entirely on withincountry variation. Simple pooled Tobit regressions find no impact of urbanization either; however, once we exclude Germany, simple pooled Tobit regressions do produce a strong negative impact of urbanization. ${ }^{29}$ This finding would not have come as a surprise to Lipset, who noted that Germany was an anomaly in the context of his theory, since it was industrialized, urbanized, well-educated, and wealthy. Once we exclude Germany from the analysis, the modernization theory seems to fit the facts better. On the other hand, simple pooled regressions omit many country-specific factors which could be relevant, which is why we prefer our baseline fixed- effects specification.

The estimates are more supportive of the hypothesis that higher effective electoral thresholds reduced votes for extremist parties; the coefficient on this variable is negative and significant in the

\footnotetext{
${ }^{28}$ As a reminder, this includes not just fascist, but also other extreme right-wing anti-system parties, although for brevity's sake we refer to "fascist" parties.

${ }^{29}$ Results available on request.
} 
TABLE 5

DETERMINANTS OF RIGHT-WING ANTI-SYSTEM VOTE SHARE, 1919-1939 (all elections)

\begin{tabular}{|c|c|c|c|c|c|c|c|c|c|}
\hline & (1) & $\begin{array}{c}(2) \\
\text { Growth over } \\
\text { Past Year }\end{array}$ & (3) & $\begin{array}{r}\text { (4) } \\
\mathrm{P} \\
\end{array}$ & $\begin{array}{c}\quad(5) \\
\text { Growth over } \\
\text { ast Two Yea }\end{array}$ & $\begin{array}{l}\text { (6) } \\
\text { ars }\end{array}$ & $\begin{array}{l}\text { (7) } \\
\mathrm{Pa} \\
\end{array}$ & $\begin{array}{c}(8) \\
\text { Growth ove } \\
\text { st Three Ye }\end{array}$ & $\begin{array}{l}\text { (9) } \\
\text { er } \\
\text { ears } \\
\end{array}$ \\
\hline \multirow[t]{2}{*}{ Growth } & $-58.79 * *$ & $-68.50 * * *$ & 13.64 & -63.39 & $-56.97^{* *}$ & -27.13 & $-109.6^{* * *}$ & $-77.32^{* * *}$ & *-79.03** \\
\hline & $(27.37)$ & (11.11) & $(13.56)$ & $(50.08)$ & $(22.20)$ & $(35.89)$ & $(39.95)$ & $(24.21)$ & $(37.51)$ \\
\hline \multirow[t]{2}{*}{ Urbanization } & & $5.013 * * *$ & -0.421 & & $6.140^{* *}$ & -0.739 & & 4.413 & -1.188 \\
\hline & & $(1.316)$ & $(1.079)$ & & $(2.462)$ & $(1.310)$ & & $(2.847)$ & $(1.277)$ \\
\hline \multirow[t]{2}{*}{$\begin{array}{l}\text { Effective } \\
\text { electoral } \\
\text { threshold }\end{array}$} & & $-0.557 * * *$ & -0.417 & & $-0.581 * * *$ & -0.303 & & $-0.532 * *$ & * -0.202 \\
\hline & & $(0.112)$ & $(0.306)$ & & $(0.183)$ & $(0.306)$ & & $(0.212)$ & $(0.200)$ \\
\hline \multirow[t]{2}{*}{ 1919-1924 } & & & $-52.12 * * *$ & & & $-52.05 * * *$ & & & $-42.15 * * *$ \\
\hline & & & $(11.91)$ & & & (11.10) & & & $(10.13)$ \\
\hline \multirow[t]{2}{*}{$1925-1928$} & & & $-20.28^{* *}$ & & & $-20.40^{* *}$ & & & -20.00 \\
\hline & & & (10.06) & & & $(8.550)$ & & & $(13.50)$ \\
\hline \multirow[t]{2}{*}{ 1929-1933 } & & & -6.605 & & & -9.309 & & & $-15.40^{* * *}$ \\
\hline & & & $(6.877)$ & & & $(6.865)$ & & & $(4.179)$ \\
\hline Observations & 148 & 148 & 148 & 136 & 136 & 136 & 125 & 125 & 125 \\
\hline
\end{tabular}

Notes: Standard errors are in parentheses. Fixed-effects panel Tobit estimators. Fixed effects not estimated by semi-parametric estimator.

Source: See the text.

specification without period dummies. ${ }^{30}$ Finally, the period dummies capture other aspects of the deteriorating political climate of the interwar period insofar as they become steadily less negative over time.

Our main interest is in the effect of GDP growth on extremism. As can be seen, GDP growth was negatively related to the vote for extreme right-wing parties. However, the effect is not robust to adding control variables and period dummies in the case of growth over one and two years. In column 3 , the coefficient on growth even turns positive. The effect is robust, on the other hand, once we look at growth over the previous three years and remains robust if we extend the horizon to four and five years. ${ }^{31}$ This is not because of falling standard errors (which are in fact increasing as we extend the growth horizon), but because of larger coefficients.

\footnotetext{
${ }^{30}$ The coefficients are however extremely small and insignificant when period dummies are introduced into the specification.

${ }^{31}$ Results not reported here.
} 
TABLE 6

DETERMINANTS OF RIGHT-WING ANTI-SYSTEM VOTE SHARE, 1919-1939

(all elections three years or more apart)

\begin{tabular}{|c|c|c|c|c|c|c|c|c|c|}
\hline & (1) & (2) & (3) & (4) & (5) & (6) & (7) & (8) & (9) \\
\hline & \multicolumn{3}{|c|}{$\begin{array}{l}\text { Growth over } \\
\text { Past Year }\end{array}$} & \multicolumn{3}{|c|}{$\begin{array}{c}\text { Growth over } \\
\text { Past Two Years }\end{array}$} & \multicolumn{3}{|c|}{$\begin{array}{c}\text { Growth over } \\
\text { Past Three Years }\end{array}$} \\
\hline \multirow[t]{2}{*}{ Growth } & -45.83 & $-67.25^{* *}$ & 12.09 & -56.21 & -51.24 & -21.97 & $-73.91^{*}$ & $-63.32^{* *}$ & $-81.25^{*}$ \\
\hline & (38.62) & (27.08) & $(29.45)$ & $(60.45)$ & (38.10) & (50.84) & $(42.02)$ & (29.36) & (45.04) \\
\hline \multirow[t]{2}{*}{ Urbanization } & & $3.777^{* * *}$ & -0.552 & & 3.883 & -1.094 & & 3.015 & $-1.468^{*}$ \\
\hline & & $(0.949)$ & (2.478) & & (2.686) & (2.110) & & (2.992) & $(0.845)$ \\
\hline \multirow[t]{2}{*}{ 1919-1924 } & & & $-50.61^{*}$ & & & $-49.66^{* * *}$ & & & $-34.63^{* * *}$ \\
\hline & & & $(26.25)$ & & & (14.39) & & & (7.428) \\
\hline \multirow[t]{2}{*}{$1925-1928$} & & & $-18.89 * *$ & & & $-19.67 * *$ & & & $-17.76^{*}$ \\
\hline & & & $(7.603)$ & & & (7.980) & & & $(9.830)$ \\
\hline \multirow[t]{2}{*}{ 1929-1933 } & & & -6.516 & & & -9.333 & & & $-16.60^{* * *}$ \\
\hline & & & (8.528) & & & $(6.731)$ & & & $(4.972)$ \\
\hline Observations & 122 & 122 & 122 & 110 & 110 & 110 & 99 & 99 & 99 \\
\hline
\end{tabular}

$* p<0.1, * * p<0.05, * * * p<0.01$.

Notes: Standard errors are in parentheses. Fixed-effects panel Tobit estimators. Fixed effects not estimated by semi-parametric estimator.

Source: See the text.

It is possible that our results might be driven by countries where more than one election fell within a particularly bad three-year period, thus allowing the same poor economic performance to count on multiple occasions in the regressions. Table 6 therefore repeats the exercise, dropping elections occurring within three years of each other. ${ }^{32}$ The effects of economic growth are very similar to those in Table $5 .{ }^{33}$ Finally, Table 7 excludes not only all elections falling within three years of each other but also all German elections. Again the results are qualitatively similar, although the growth coefficients are smaller, as expected, and the coefficient on three-year growth only becomes statistically significant when period dummies are included. ${ }^{34}$

\footnotetext{
${ }^{32}$ The procedure we follow is to include in our sample the first election in any country, and then move forwards in time to include the next election falling outside the three-year window, repeating the procedure until the end of the period.

${ }^{33}$ The pantob procedure does not solve properly for this smaller sample when the electoral threshold variable is included, which is why we exclude it from the specifications in Table 6.

${ }^{34}$ Note that the pantob procedure did not solve properly when the first period dummy variable was included, in cases where Germany was excluded from the sample. This variable is therefore excluded from the regressions in Table 7.
} 
TABLE 7

DETERMINANTS OF RIGHT-WING ANTI-SYSTEM VOTE SHARE, 1919-1939

(all non-German elections three years or more apart)

\begin{tabular}{|c|c|c|c|c|c|c|c|c|c|}
\hline & (1) & $\begin{array}{c}(2) \\
\text { Growth ove } \\
\text { Past Year }\end{array}$ & er & $\begin{array}{l}\text { (4) } \\
\mathrm{Pc} \\
\end{array}$ & $\begin{array}{l}\text { (5) } \\
\text { Srowth ove } \\
\text { st Two Ye }\end{array}$ & $\begin{array}{l}\text { (6) } \\
\text { ars }\end{array}$ & $\begin{array}{l}\text { (7) } \\
\mathrm{Pa} \\
\end{array}$ & $\begin{array}{c}\quad(8) \\
\text { Growth ove } \\
\text { st Three Ye }\end{array}$ & $\begin{array}{l}\text { (9) } \\
\text { er } \\
\text { ears }\end{array}$ \\
\hline \multirow[t]{2}{*}{ Growth } & -21.70 & -27.35 & -26.36 & -7.717 & -7.318 & -7.639 & -28.11 & -21.87 & $-57.63^{*}$ \\
\hline & (28.87) & (21.67) & $(30.72)$ & (34.11) & (12.64) & (19.95) & (19.50) & (24.18) & $(34.80)$ \\
\hline \multirow[t]{2}{*}{ Urbanization } & & 2.044 & 1.474 & & 1.558 & 0.882 & & 1.580 & 0.518 \\
\hline & & (1.695) & $(1.212)$ & & (1.321) & (1.076) & & (1.932) & (1.802) \\
\hline \multirow[t]{2}{*}{ 1925-1928 } & & & -7.570 & & & -7.231 & & & -4.363 \\
\hline & & & (9.589) & & & (8.819) & & & (5.686) \\
\hline \multirow[t]{2}{*}{ 1929-1933 } & & & -0.996 & & & -1.845 & & & -8.777 \\
\hline & & & $(7.175)$ & & & (7.444) & & & $(6.671)$ \\
\hline Observations & 117 & 117 & 117 & 106 & 106 & 106 & 96 & 96 & 96 \\
\hline
\end{tabular}

Notes: Standard errors are in parentheses. Fixed-effects panel Tobit estimators. Fixed effects not estimated by semi-parametric estimator.

Source: See the text.

The results suggest that it was growth over a prolonged period (three years or more), rather than growth over the preceding year alone, that really made a difference for the fascist vote. This makes intuitive sense. Societies can generally weather even large one-off shocks. But when economic bad news continues beyond a certain period of time and negative expectations become firmly entrenched, some people reach for extreme solutions.

While as economic historians we are interested in the sizes of these effects, the semi-parametric fixed-effects Tobit estimator does not allow us to compute these. We therefore also estimated fixedeffects Tobit models using a maximum likelihood estimator to gain some sense of the magnitudes involved. ${ }^{35}$ For example, the simple model in column 7 of Table 5 (where the fascist vote is related to growth over the previous three years, with no other control variables being added to the specification) yielded a coefficient on three-year growth of -37.08 , statistically significant at the 1 percent level. A J. F. McDonald and R. A. Moffitt (1980) decomposition implies a marginal effect of growth on the fascist vote share of -13.87 in

\footnotetext{
${ }^{35}$ Implemented with Limdep, this estimator estimates country-fixed effects by "brute force." Greene (2004) argues that the incidental parameters problem is overstated in the context of this model. The results are reported in the working paper version of this article.
} 
countries where this vote was nonzero: a one-standard-deviation decline in growth $(0.1265)$ is associated with an increase in the fascist vote share of 1.75 percent.

The point estimates imply that the deterioration in growth in Germany between $1928(0.181)$ and $1932(-0.158)$ is associated with an increase in the fascist vote of 4.7 percent. This compares with a total increase of 40 percentage points (or 19 percentage points if the DVP and DNVP are classified as fascist throughout). Evidently, then, our results so far can only explain a limited share of the increase in the right-wing anti-system vote in cases like Germany between 1928 and 1932.

Similarly, the estimate yields a marginal impact of growth on the probability of there being a fascist party in the first place of -1.45 . This implies that a one-standard-deviation increase in growth lowers the probability of there being a fascist party by 18 percent. ${ }^{36}$

We saw evidence earlier that the extent to which the fascist vote increased after 1929 depended on country characteristics. Did the impact of economic growth depend on these same factors? To explore this possibility, we estimated regressions similar to those in Tables 5-7, but interacting growth with the country-specific variables in Table 4. The only interaction effect that is robust to excluding Germany is that involving a history of prewar democracy.

Table 8 reports regressions in which both growth and its interaction with prewar democracy appear on the right-hand side. ${ }^{37}$ Column 1 gives the results obtained when using all available observations; column 2 excludes all elections occurring within three years of each other; and column 3 excludes both the German observations and all elections occurring within three years of each other. Table 8 shows that while growth had a large impact on voting for right-wing extremist parties in countries which had not been democratic prior to World War I, a history of prewar democracy largely eliminates this effect. That is to say, the interaction term between growth and prewar democracy is positive, almost as large in absolute terms as the negative coefficient measuring the direct impact of growth, and statistically significant.

\footnotetext{
${ }^{36}$ Note that this last statement is about the probability that the vote will be positive (i.e., nonzero, i.e., that a fascist party will exist in the first place); the 1.75 percent rise referred to earlier is a statement about the vote share of fascist parties, given that they already existed.

${ }^{37}$ It excludes urbanization from the basic specification since the performance of this variable is disappointing throughout. Note that once again, excluding elections falling within three years of each other meant that the model did not solve properly when the electoral threshold was included; it was thus dropped from the regressions in columns 2 and 3.
} 
TABLE 8

DETERMINANTS OF RIGHT-WING ANTI-SYSTEM VOTE SHARE, 1919-1939

(1)

\begin{tabular}{lccc} 
Sample & All & $\begin{array}{c}\text { All Elections Three } \\
\text { Years or More Apart }\end{array}$ & $\begin{array}{c}\text { All Non-German } \\
\text { Elections Three } \\
\text { Years or More Apart }\end{array}$ \\
\hline Growth over past three years & $-97.58^{* * *}$ & $-103.9^{* * *}$ & $-50.58^{* *}$ \\
& $(24.67)$ & $(29.19)$ & $(23.22)$ \\
Growth*prewar democracy & $83.38^{* * *}$ & $86.98^{* * *}$ & $37.03^{*}$ \\
Effective electoral threshold & $(26.71)$ & $(29.23)$ & $(22.45)$ \\
& -0.224 & & \\
$1919-1924$ & $(0.164)$ & & $-18.36^{* * *}$ \\
& $-31.50^{* * *}$ & $-23.31 * * *$ & $(6.386)$ \\
$1925-1928$ & $(7.767)$ & $(7.688)$ & -8.708 \\
$1929-1933$ & -12.94 & -10.53 & $(8.999)$ \\
& $(9.913)$ & $(9.389)$ & $-11.00^{*}$ \\
Observations & $-12.08^{*}$ & $-13.23 *$ & $(6.277)$ \\
\hline
\end{tabular}

$* p<0.1, * * p<0.05, * * * p<0.01$.

Notes: Standard errors are in parentheses. Fixed-effects panel Tobit estimators. Fixed effects not estimated by semi-parametric estimator. Sample sizes vary. (1) all observations; (2) all observations excluding those occurring within three years of each other; (3) all non-German elections excluding those occurring within three years of each other.

Source: See the text.

Again, we also estimated a fixed-effects Tobit model using MLE methods in order to gauge the magnitude of these effects. Estimating a model equivalent to that in column 1 implies a coefficient on growth of -78.45 and a coefficient on the interaction term of 67.82 , both statistically significant at the 1 percent level. The results indicate that in countries without a prewar history of democracy and with a preexisting fascist vote, a one-standard-deviation increase in growth is associated with a decline in the extreme right-wing vote share of 2.9 percent. In such countries, a decline in growth on the order of that experienced by Germany between 1928 and 1932 is associated with an increase in the fascist vote share of 7.8 percent. This is over 40 percent of the increase in the extreme right-wing anti-system vote share observed in Germany in the period if one adopts the alternative definition of the extreme right-wing vote in Germany mentioned earlier (DVP + DNVP + NSDAP). In countries that had not been democratic prior to 1914, 
a one-standard-deviation rise in growth is associated with a 58 percent decline in the probability of observing a positive fascist vote. These are large effects.

There are three important qualifications to the conclusion that economic growth was bad for extremists. First, there is no evidence of a growth effect on the Communist vote. ${ }^{38}$ Regression analysis thus strengthens our earlier finding that the Great Depression, the crisis of capitalism par excellence, benefited the extreme right but not the extreme left.

The shift in this period towards strident sectarianism by the Communist movement helps to explain this apparent paradox. Following the Third International's Sixth Congress in 1928, the "Left Turn" "demanded that Communist parties become the sole anti-capitalist rallying points, destroy reformist illusions in the working class and oppose all cooperation with social democrats, who became the main enemy. This last instruction, denouncing social democrats through the infamous formula of 'Social Fascism,' repudiated coalition building in the working class" (Eley 2002, p. 252). Most observers have agreed with E. Hobsbawm's (1994, p. 71) assessment that this strategy was "politically calamitous." To make matters worse, many Social Democratic parties were still in thrall to pre-Leninist orthodox Marxist pieties, waiting for the collapse of capitalism rather than seeking its reform (Berman 2006, 2008). In sharp contrast, national socialists and fascists were promising active intervention in the economy to improve peoples' lives. "Because of their emphasis on letting economic forces be the drivers of history, orthodox Marxists...ceded ground to activist groups on the right" (Berman 2006, p. 97).

The second important qualification is that short-lived economic fluctuations did not have a major impact on the extremist right-wing vote. To repeat, it was longer-run business cycle conditions that mattered.

Third, the link between the fascist vote and the business cycle was not the same everywhere. That link was especially close where the absence of a long-standing democratic tradition made the political soil fertile for those seeking to undermine the existing system. In contrast, countries with longer histories of democracy seem to have been largely immune from fascism, and the link between economic growth and extremist voting behavior was correspondingly weaker. As Hobsbawm (1994, p. 138) puts it, "in well-established and stable democracies the very process of regular voting has tended to give

\footnotetext{
${ }^{38}$ These additional regressions, not reported here, are available on request.
} 
citizens - even those in the minority - a sense that the electoral process legitimizes the governments it produces. But few of the interwar democracies were well-established."

\section{ROBUSTNESS}

Table 9 provides some robustness checks. Column 1 shows the baseline specification, as in column 9 of Tables 5-7. Column 2 shows that the negative impact of growth on the fascist vote survives when a random effects Tobit estimator is used instead of the fixed-effects estimator. Column 3 replaces the period dummies which we have used thus far with year dummies; again, the results continue to go through, although the effect is statistically insignificant when the sample is reduced in Panels B and C. Column 4 shows that the results still hold when we use the standard fixed-effects OLS estimator with year dummies and standard errors clustered by country. Column 5 tests whether the impact of growth is nonlinear by introducing a squared growth term. The coefficient on this term is negative but statistically insignificant in Panel A; it becomes bigger in absolute terms and statistically significant in Panels B, and especially C. ${ }^{39}$

Some readers may worry that we have not identified a causal relationship, since rising political extremism could scare away investment, and cause recessions to deepen. Another possibility is that a fear of fascism might have led governments to behave in ways that worsened the depression, although insofar as they responded to political radicalization by moderating austerity and loosening purse strings this would have lessened the economic downturn: multipliers were very high during this period, of the order of 1.6, or even higher (Almunia et al. 2010). The fact that it is cumulative growth over the three previous years that is robustly related to the fascist vote, rather than growth in the previous period alone, should help to allay such fears. So should the finding, in column 6 , that our results are robust to lagging our three-year growth variable by one year. No one to our knowledge has argued that the Great Depression, which was a worldwide phenomenon, was caused by investors' rational anticipation of the political consequences that would ensue if it occurred.

\footnotetext{
${ }^{39}$ We also tried estimating models in which the impact of growth varied depending on whether growth was positive or negative. The results were inconclusive, perhaps because in the smaller samples of Panels B and C there were not enough data-points available to make such an exercise a reliable one.
} 
TABLE 9 DETERMINANTS OF RIGHT-WING ANTI-SYSTEM VOTE SHARE, 1919-1939

(1)

(2)

(3)

(4)

(5)

(6)

Semi- RE Tobit, RE Tobit, FE OLS, Semi- SemiParametric Period Year Year Parametric Parametric Model FE Tobit Dummies Dummies Dummies FE Tobit FE Tobit

Panel A. Entire sample

\begin{tabular}{|c|c|c|c|c|c|c|}
\hline \multirow[t]{2}{*}{$\begin{array}{l}\text { Effective electoral } \\
\text { threshold }\end{array}$} & -0.202 & -0.311 & -0.224 & -0.131 & -0.190 & -0.246 \\
\hline & $(0.200)$ & $(0.221)$ & $(0.227)$ & $(0.129)$ & $(0.178)$ & $(0.235)$ \\
\hline \multirow[t]{2}{*}{ Urbanization } & -1.188 & -0.216 & -0.232 & 0.0434 & -0.919 & -2.926 \\
\hline & $(1.277)$ & $(0.252)$ & $(0.246)$ & $(0.159)$ & $(2.423)$ & $(2.092)$ \\
\hline \multicolumn{6}{|l|}{ Growth over past } & \\
\hline & $(37.51)$ & $(13.55)$ & $(14.20)$ & $(5.701)$ & $(49.74)$ & \\
\hline \multirow[t]{2}{*}{$\begin{array}{l}\text { Growth over past } \\
\text { three years squared }\end{array}$} & & & & & -200.4 & \\
\hline & & & & & $(237.6)$ & \\
\hline \multirow[t]{2}{*}{$\begin{array}{l}\text { Growth over past } \\
\text { three years lagged }\end{array}$} & & & & & & $-81.93 * * *$ \\
\hline & & & & & & $(23.01)$ \\
\hline \multirow[t]{2}{*}{ Constant } & & $16.28 *$ & -4.728 & 2.319 & & \\
\hline & & $(8.702)$ & (11.44) & $(2.693)$ & & \\
\hline Observations & 125 & 125 & 125 & 125 & 125 & 119 \\
\hline
\end{tabular}

Panel B. Excluding elections falling within three years of each other

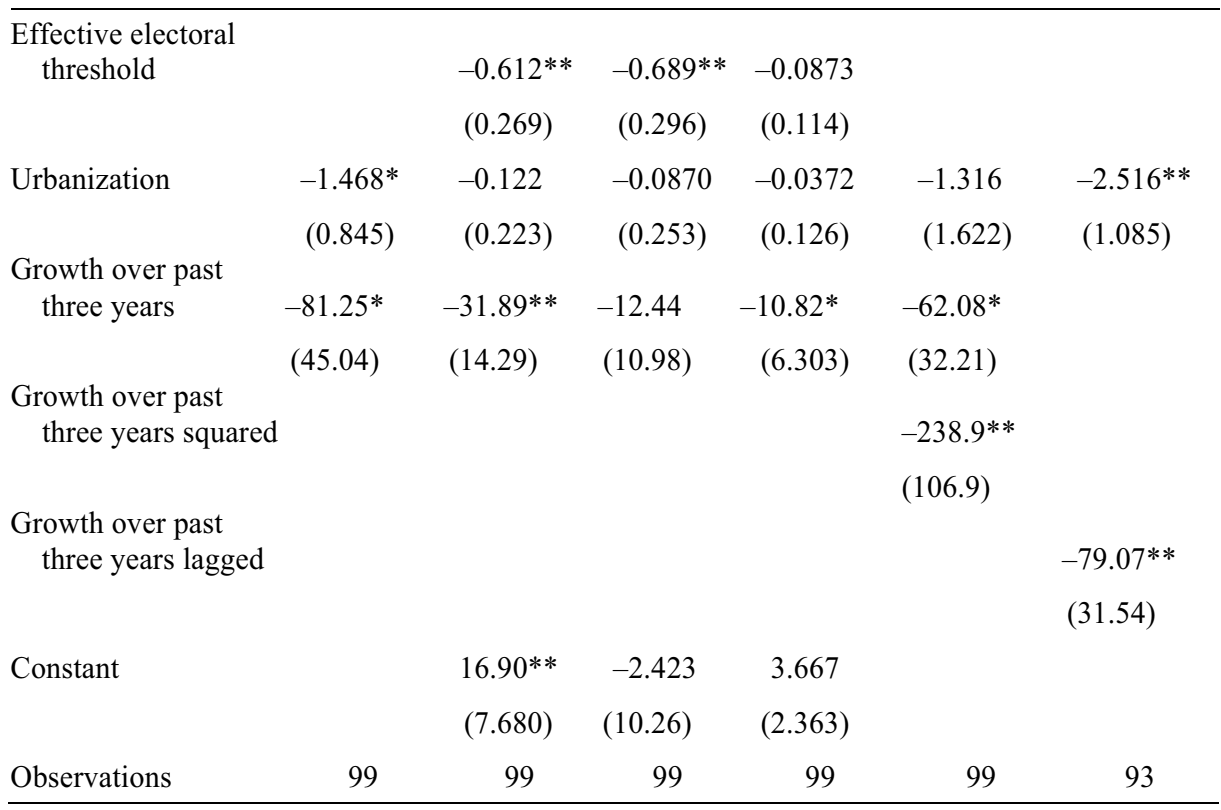


TABLE $9-$ continued

\begin{tabular}{|c|c|c|c|c|c|c|}
\hline & (1) & (2) & (3) & (4) & (5) & (6) \\
\hline Model & $\begin{array}{c}\text { Semi- } \\
\text { Parametric } \\
\text { FE Tobit }\end{array}$ & $\begin{array}{l}\text { RE Tobit, } \\
\text { Period } \\
\text { Dummies }\end{array}$ & $\begin{array}{l}\text { RE Tobit, } \\
\text { Year } \\
\text { Dummies }\end{array}$ & $\begin{array}{c}\text { FE OLS, } \\
\text { Year } \\
\text { Dummies }\end{array}$ & $\begin{array}{l}\text { Semi- } \\
\text { Parametric } \\
\text { FE Tobit }\end{array}$ & $\begin{array}{l}\text { Semi- } \\
\text { Parametric } \\
\text { FE Tobit }\end{array}$ \\
\hline \multicolumn{7}{|c|}{ Panel C. Excluding elections falling within three years of each other and all German elections } \\
\hline $\begin{array}{l}\text { Effective electoral } \\
\text { threshold }\end{array}$ & & $\begin{array}{l}-0.264 \\
(0.199)\end{array}$ & $\begin{array}{c}-0.188 \\
(0.186)\end{array}$ & $\begin{array}{c}0.00243 \\
(0.0564)\end{array}$ & & \\
\hline Urbanization & $\begin{array}{c}0.518 \\
(1.802)\end{array}$ & $\begin{array}{c}-0.406^{* *} \\
(0.192)\end{array}$ & $\begin{array}{c}-0.464 * * * \\
(0.175)\end{array}$ & $\begin{array}{c}-0.133 * * \\
(0.0667)\end{array}$ & $\begin{array}{c}-0.979 \\
(0.928)\end{array}$ & $\begin{array}{l}-0.533 \\
(1.660)\end{array}$ \\
\hline $\begin{array}{l}\text { Growth over past } \\
\text { three years }\end{array}$ & $\begin{array}{l}-57.63^{*} \\
(34.80)\end{array}$ & $\begin{array}{c}-21.49 * \\
(12.28)\end{array}$ & $\begin{array}{r}-13.78 \\
(9.799)\end{array}$ & $\begin{array}{l}-7.532 * \\
(4.203)\end{array}$ & $\begin{array}{r}0.261 \\
(41.29)\end{array}$ & \\
\hline $\begin{array}{l}\text { Growth over past } \\
\text { three years squared }\end{array}$ & & & & & $\begin{array}{l}-428.2^{* * *} \\
(137.3)\end{array}$ & \\
\hline $\begin{array}{l}\text { Growth over past } \\
\text { three years lagged }\end{array}$ & & & & & & $\begin{array}{c}-57.56 * * \\
(25.42)\end{array}$ \\
\hline Constant & & $\begin{array}{c}20.58 * * * \\
(6.101)\end{array}$ & $\begin{array}{c}5.430 \\
(6.635)\end{array}$ & $\begin{array}{l}3.947 * * \\
(1.576)\end{array}$ & & \\
\hline Observations & 96 & 96 & 96 & 96 & 96 & 90 \\
\hline
\end{tabular}

$* p<0.1, * * p<0.05, * * * p<0.01$.

Notes: Standard errors are in parentheses. Fixed-effects not estimated by semi-parametric estimator. Period and year dummies not reported.

Source: See the text.

Nonetheless, it is clearly possible that our correlations may in part be capturing the toxic feedback loop between a deteriorating economy and a worsening political climate. Insofar as this is the case, it strengthens the argument that better macroeconomic policymaking early in the crisis would have paid rich dividends.

One notable feature of the results in Table 9 is that the coefficient on the electoral threshold is negative in all but one case, and is statistically significant in two. A second is that the coefficient on the urbanization variable is also negative in all but one case, and is statistically significant in five. While we prefer our baseline specification, there is thus some support for the Lipset modernization thesis here, as well as for the argument that electoral systems mattered. 
TABLE 10

DETERMINANTS OF EXTREMIST SEAT SHARES, 1919-1939

Extremist Parties

$\frac{\text { Sample }}{\begin{array}{c}\text { Vote share of extremist } \\ \text { party }\end{array}}$

Effective electoral

threshold

1919-1924

1925-1928

1929-1933

Observations
(1)

(2)

Right-Wing Anti-System

All Excluding

All Germany

$1.220^{* * *}$

(0.0606)

(0.0375)

$-0.129 * * *$
$(0.0192)$

0.761

(0.617)

0.392

(0.575)

0.330

$(0.968)$

159
(3)

(4)

$* p<0.1, * * p<0.05, * * * p<0.01$.

Notes: Standard errors are in parentheses. Fixed-effects panel Tobit estimators. Fixed effects not estimated by semi-parametric estimator.

Source: See the text.

Finally, how did votes for extremist parties translate into seats? The more votes a party got, the more seats it got, other things equal, but in practice the relevant other things could vary. Table 10 presents fixed-effects panel Tobit regressions of seat shares as a function of vote shares and the effective electoral threshold. It shows that higher electoral thresholds lowered the seat shares of extremist parties, both on the right and the left, with the effect being particularly pronounced for Communist parties (consistent with the evidence in column 7 of Table 4). The results are strong and robust to excluding Germany. They help to explain why we earlier found that higher electoral thresholds lowered electoral support for fascists and why proportional representation increased the Communist vote. Voters might have been unwilling to "waste" their ballots by voting for candidates who were unlikely to gain seats in parliament. In addition, when the crisis struck, extremists had fewer seats in parliament in countries with higher electoral thresholds. This mattered, since a preexisting parliamentary party could help in galvanizing support when the crisis began. The fascist vote rose on average by 1.3 percentage points after 1929 in countries where fascists had no previous parliamentary representation. 
By comparison, it rose on average by 7.5 percentage points in countries where they had already entered parliament.

There was therefore a role for political institutions in determining the relative fate of extremist parties across countries during the interwar period.

\section{CONCLUSIONS}

Any explanation for interwar political extremism must start with World War I, which cast a shadow over the decades that followed. The support that social democratic parties lent to governments in that terrible conflict alienated workers, especially in countries on the losing side. Support for the emerging communist movement was one response. Speaking of Germany, Eric Weitz (1997, p. 63) writes that "The Social Democratic party and the trade unions, collaborators in the war effort, found their claims to representation challenged by their own constituents...disastrous wartime conditions radicalized a substantial number of workers, and the great upsurge of strikes, demonstrations, and armed revolutions - from Russia on across the continent-seemed to herald the new society." It is no surprise that the communist vote was higher after 1919 in defeated than victorious countries.

In addition to radicalizing working-class politics and boosting the communist vote in defeated countries, the war and the Russian revolution it spawned splintered the left, with increasingly bitter divisions emerging between old social democratic parties on the one hand and new communist parties on the other. It "marked the beginning of the organizational divisions of the socialist movement. The results of almost half a century of efforts to organize, unify, and centralize socialist movements suddenly melted away almost everywhere between 1910 and 1920" (Bartolini 2000, p. 87). Sectarian tensions on the left increased further in 1928, coincident with the onset of a worldwide economic slump that demanded an activist response. That response, as a result, did not by and large come from the left, with communists focused on attacking "social fascism" and many social democrats often unable or unwilling to offer radical policy responses to increasingly frustrated voters. ${ }^{41}$ Instead, it was fascist parties that seemingly offered a "Third Way" between communism and liberal economic orthodoxy. It was fascists who

\footnotetext{
${ }^{40}$ Regression results are available on request.

${ }^{41}$ Only in countries like Sweden, where Social Democracy had evolved to the point where it closely resembled what "Social Democracy" would come to mean after 1945, and where Social Democratic parties promoted activist economic policies, did the left reap substantial electoral rewards (Berman 2006).
} 
benefited electorally after 1929 . They benefited most in countries that were defeated in 1918 and where the electorate had been radicalized: here communism gained after 1919, and fascism after 1929. They benefited particularly in countries that were least inoculated against the virus of political extremism by long experience with democracy.

It was fascists therefore who benefitted politically from poor economic conditions. Cumulative growth was negatively related to the fascist vote, even after controlling for country- and period-specific conditions. The link was particularly tight in countries that were not democratic before 1914. This last pattern is consistent with the conclusion of Almond and Verba (1989) that political culture matters, and with the argument of Persson and Tabellini (2009) that countries with longer histories of democracy accumulate social and political capital increasing the likelihood that they will be able to resist threats to the prevailing political system.

Finally, the success of anti-system parties was shaped by the structure of the electoral system. Predictably, a higher minimum share of the vote needed for a party to gain parliamentary representation made it more difficult for extremists to gain seats in parliament. Less obviously, a higher threshold also appears to have lowered votes for extremists in the first place.

Not all aspects of Germany's unfortunate political history in this period generalize. But some do. That a country had been on the losing side in World War I, had limited democratic capital, possessed an electoral system conducive to entry and, above all, had a serious economic slump made it more likely that right-wing anti-system parties would gain a political foothold not just in Germany but more widely. 


\section{Appendix}

\begin{tabular}{|c|c|}
\hline & $\begin{array}{c}\text { APPENDIX TABLE } 1 \\
\text { PARTIES CLASSIFIED AS ANTI-SYSTEM } \\
\end{array}$ \\
\hline Argentina & Communist Party (C) \\
\hline Australia & Communist Party of Australia (C) \\
\hline Austria & $\begin{array}{l}\text { German Nationalists (S), Greater German's People's Party (S), Heimatbloc (F), } \\
\text { National Socialists - NSDAP (F), Communist Party (C) }\end{array}$ \\
\hline Belgium & Rexists (F), Flemish Nationalists (S/F), Communist Party (C) \\
\hline Bulgaria & Communists (C) \\
\hline Canada & Communist Party (C) \\
\hline Chile & PCCh - Communists (C), Partido Nacista (F) \\
\hline Czechoslovakia & $\begin{array}{l}\text { DNSAP (F) - 1920-1933, DNP (F) - 1920-1933, BdL (S) 1920-1922, DCVP } \\
\text { (S) - 1920-1922, DSAP (S) - 1920-1922, } \\
\text { SdP (F) - 1933-1938,KSC (C) - 1921-1938, NOF (F) - 1929-1938, NS (F) - } \\
\text { 1935-1938, Smaller Ethnic Parties (S) - 1920-1938. }\end{array}$ \\
\hline Denmark & Communists (C), National Socialists (F) \\
\hline Finland & $\begin{array}{l}\text { National Coalition (F) (1929-1935), Patriotic People's Movement (F) (1933- } \\
\text { 1939), Communists under the Socialist Worker's Party (C) (excluded from } 1930 \\
\text { onwards) }\end{array}$ \\
\hline France & Communists (C), Independents Right Wing (F) \\
\hline Germany & $\begin{array}{l}\text { National Socialists (F), Communists (C), National People's Party (F) - 1928- } \\
1933\end{array}$ \\
\hline Greece & Communists (C), Free Thinkers' Party (F) (Metaxas) \\
\hline Hungary & $\begin{array}{l}\text { Arrow Cross (F), National Socialists (F), Hungarian National Independence Party } \\
\text { (F), Extreme right Independents and smaller parties (F) }\end{array}$ \\
\hline Ireland & Communists (C) \\
\hline Italy & Communists (C), Fascists (F), Ex-Servicemen Party (F) \\
\hline The Netherlands & Communist Party (C), National Socialist Party (F) \\
\hline New Zealand & Communist Party (C) \\
\hline Norway & Communist Party (C), National Socialist Party (F) \\
\hline Poland & Communist Party $(\mathrm{C})$ \\
\hline Romania & $\begin{array}{l}\text { Iron Guard (F), League of National Christian Defence (F), National Christian } \\
\text { Party (F) }\end{array}$ \\
\hline Spain & $\begin{array}{l}\text { Communists (C), Marxist POUM (C), Falange (F), Bloque Nacional (F), Catalan } \\
\& \text { Basque Nationalists (S) }\end{array}$ \\
\hline Sweden & Communists (C), National Socialists (F) \\
\hline Switzerland & Communists (C), Front Party (F) \\
\hline United Kingdom & Communist Party (C) \\
\hline United States & No electorally successful parties identified \\
\hline Uruguay & Communist Party (C) \\
\hline Yugoslavia & Communist Party (C), Croatian People's (Republican) Peasant Party (S) 1919-1925 \\
\hline
\end{tabular}

$(\mathrm{F})=$ Extreme Right-Wing Parties; $(\mathrm{C})=$ Communist Parties; $(\mathrm{S})=$ Secessionist Parties

Sources: Party classifications are from Capoccia (2005), or are based on the criteria outlined

in Capoccia $(2002,2005)$ using Payne (1996) and various country histories. 
APPENDIX TABLE 2

VALUES OF COUNTRY-SPECIFIC DUMMY VARIABLES

\begin{tabular}{|c|c|c|c|c|c|c|c|c|}
\hline & $\begin{array}{c}\text { Prewar } \\
\text { Democracy }\end{array}$ & $\begin{array}{c}\text { Pre-1929 } \\
\text { Fascist } \\
\text { Seat } \\
\end{array}$ & $\begin{array}{c}\text { Agricultural } \\
\text { Elite }\end{array}$ & $\begin{array}{l}\text { Religious } \\
\text { Divide }\end{array}$ & $\begin{array}{l}\text { Ethnic/ } \\
\text { Ling. } \\
\text { Divide } \\
\end{array}$ & $\begin{array}{l}\text { WWI } \\
\text { Loser } \\
\end{array}$ & $\begin{array}{c}\text { Boundary } \\
\text { Change }\end{array}$ & $\begin{array}{c}\text { Above } \\
\text { Median } \\
\text { Urban }\end{array}$ \\
\hline Argentina & YES & NO & YES & NO & NO & NO & NO & YES \\
\hline Australia & YES & NO & YES & YES & NO & NO & NO & YES \\
\hline Austria & NO & NO & YES & NO & NO & YES & YES & YES \\
\hline Belgium & YES & NO & NO & NO & YES & NO & YES & SOMETIMES \\
\hline Bulgaria & NO & NO & NO & YES & YES & YES & YES & NO \\
\hline Canada & YES & NO & NO & YES & YES & NO & NO & YES \\
\hline Chile & YES & NO & YES & NO & NO & NO & NO & SOMETIMES \\
\hline Czechoslovakia & NO & YES & YES & YES & YES & YES & YES & NO \\
\hline Denmark & YES & NO & NO & NO & NO & NO & YES & SOMETIMES \\
\hline Finland & NO & YES & NO & NO & NO & NO & NO & NO \\
\hline France & YES & YES & NO & NO & NO & NO & YES & SOMETIMES \\
\hline Germany & NO & YES & YES & YES & NO & YES & YES & YES \\
\hline Greece & NO & YES & NO & NO & NO & NO & YES & NO \\
\hline Hungary & NO & YES & YES & NO & NO & YES & YES & SOMETIMES \\
\hline Ireland & NO & NO & NO & NO & NO & NO & YES & NO \\
\hline Italy & NO & YES & YES & NO & NO & NO & YES & SOMETIMES \\
\hline The Netherlands & YES & NO & NO & YES & NO & NO & NO & YES \\
\hline New Zealand & YES & NO & YES & YES & YES & NO & NO & YES \\
\hline Norway & YES & NO & NO & NO & NO & NO & NO & NO \\
\hline Poland & NO & NO & YES & YES & YES & NO & YES & NO \\
\hline Romania & NO & YES & YES & NO & YES & NO & YES & NO \\
\hline Spain & NO & NO & YES & NO & YES & NO & NO & SOMETIMES \\
\hline Sweden & YES & NO & NO & NO & $\mathrm{NO}$ & NO & $\mathrm{NO}$ & NO \\
\hline Switzerland & YES & NO & NO & YES & YES & NO & NO & NO \\
\hline United Kingdom & YES & NO & YES & NO & $\mathrm{NO}$ & NO & YES & YES \\
\hline United States & YES & NO & NO & YES & YES & NO & NO & YES \\
\hline Uruguay & NO & NO & YES & NO & NO & NO & NO & SOMETIMES \\
\hline Yugoslavia & NO & NO & NO & YES & YES & NO & YES & NO \\
\hline
\end{tabular}

Notes: The dummy variable indicating whether a country has an urbanization rate higher than the sample median ( 29.5 percent) is time-varying in the countries marked "sometimes," since their urbanization rates were sometimes below and sometimes above this level. The other variables are time-invarying.

Sources: Listed in the text. 


\section{REFERENCES}

Acemoglu, D., and J. A. Robinson. Economic Origins of Dictatorship and Democracy. New York: Cambridge University Press, 2006.

Almond, G. A., and S. Verba. The Civic Culture: Political Attitudes and Democracy in Five Nations. London: Sage, 1989 [first edition 1963].

Almunia, M., Bénétrix, A., Eichengreen, B., O’Rourke, K. H. and Rua, G. "From Great Depression to Great Credit Crisis: Similarities, Differences, and Lessons." Economic Policy 25, no. 62 (2010): 219-65.

Banks, A. S. "Cross-National Time-Series Data Archive." Databanks International, Jerusalem, Israel. Available at http://www.databanksinternational.com (2011).

Bartolini, S. The Political Mobilization of the European Left, 1860-1980: The Class Cleavage. Cambridge: Cambridge University Press, 2000.

Berman, S. The Primacy of Politics: Social Democracy and the Making of Europe's Twentieth Century. Cambridge: Cambridge University Press, 2006. . "Taming Extremist Parties: Lessons from Europe." Journal of Democracy 19, no. 1 (2008): 5-18.

Bermeo, N. Ordinary People in Extraordinary Times: The Citizenry and the Breakdown of Democracy. Princeton, NJ: Princeton University Press, 2003.

Berg-Schlosser, D., and J. Mitchell. Conditions of Democracy in Europe, 1919-39: Systematic Case Studies. New York: St. Martin's Press, 2000.

. Authoritarianism and Democracy in Europe, 1919-1939: Comparative Analyses. New York: Palgrave Macmillan, 2002.

Boemeke, M. F. The Treaty of Versailles: A Reassessment After 75 Years. Cambridge: Cambridge University Press, 1998.

Boix, C. "Setting the Rules of the Game: The Choice of Electoral Systems in Advanced Democracies." American Political Science Review 93, no. 3 (1999): 609-24.

Borchardt, K. Perspectives on Modern German Economic History and Policy. Cambridge: Cambridge University Press, 1991.

Capoccia, G. “Anti-System Parties: A Conceptual Reassessment." Journal of Theoretical Politics 14, no. 1 (2002): 9-35.

. Defending Democracy: Reactions to Extremism in Interwar Europe. Baltimore, MD: Johns Hopkins University Press, 2005.

Colomer, J. M. Handbook of Electoral System Choice. New York: Palgrave Macmillan, 2004.

Czech Statistical Office. Available at www.czso.cz/csu/2006edicniplan.nsf/publ/421906-1920_az_1935 (accessed 2010).

Diamond, L. Developing Democracy: Toward Consolidation. Baltimore, MD: Johns Hopkins University Press, 1999.

Eley, G. Forging Democracy: The History of the Left in Europe, 1850-2000. Oxford: Oxford University Press, 2002.

Frey, B. S., and H. Weck. "A Statistical Study of the Effect of the Great Depression on Elections: The Weimar Republic, 1930-1933." Political Behavior 5, no. 4 (1983): 403-20.

Gerrits, A. W. M., and D. J. Wolffram. Political Democracy and Ethnic Diversity in Modern European History. Stanford, CA: Stanford University Press, 2005.

Golder, M. "Explaining Variation in the Success of Extreme Right Parties in Western Europe.” Comparative Political Studies 36, no. 4 (2003): 432-66.

Greene, W. "Fixed Effects and Bias Due to the Incidental Parameters Problem in the Tobit Model." Econometric Reviews 23, no. 2 (2004): 125-47. 
Hermens, F. A. Democracy or Anarchy? A Study of Proportional Representation. Notre Dame: University of Notre Dame Press, 1941.

Hobsbawm, E. The Age of Extremes: 1914-1991. London: Michael Joseph, 1994. (Page numbers refer to the 1995 Abacus paperback edition.)

Holzer, J. "The Heritage of the First World War." In Authoritarianism and Democracy in Europe, 1919-1939: Comparative Analyses, edited by D. Berg-Schlosser and J. Mitchell, 7-38. New York: Palgrave Macmillan, 2002.

Honoré, B. "Trimmed Lad and Least Squares Estimation of Truncated and Censored Regression Models with Fixed Effects," Econometrica 60, no. 3 (1992): 533-65.

Ivanov, M. Bulgarian GDP, 1870-1945. Forthcoming.

Jackman, R., and K. Volpert. "Conditions Favoring Parties of the Extreme Right in Western Europe." British Journal of Political Science 26, no. 4 (1996): 501-21.

James, H., and E. Müller-Luckner. The Interwar Depression in an International Context. Munich: Oldenbourg Wissenschaftsverlag, 2002.

Karvonen, L., and S. Quenter. "Electoral Systems, Party System Fragmentation, and Government Instability." In Authoritarianism and Democracy in Europe, 19191939: Comparative Analyses, edited by D. Berg-Schlosser and J. Mitchell, 13162. New York: Palgrave Macmillan, 2002.

King, G., O. Rosen, M. Tanner, and A. F. Wagner. "Ordinary Economic Voting Behavior in the Extraordinary Election of Adolf Hitler." The Journal of Economic History 68, no. 4 (2008): 951-96.

Knigge, P. "The Ecological Correlates of Right-Wing Extremism in Western Europe." European Journal of Political Research 34, no. 2 (1998): 249-79.

Kocka, J. "German History Before Hitler: The Debate About the German Sonderweg." Journal of Contemporary History 23, no. 1 (1988): 3-16.

Lijphart, A. Electoral Systems and Party Systems: A Study of Twenty-Seven Democracies, 1945-1990. New York: Oxford University Press, 1994.

Linz, J. L., and A. Stepan, eds. The Breakdown of Democratic Regimes. Baltimore, MD: Johns Hopkins University Press, 1978.

Lipset, S. M. "Some Social Requisites of Democracy: Economic Development and Political Legitimacy." American Political Science Review 53, no. 1 (1959): 69-105.

Luebbert, G. M. "Social Foundations of Political Order in Interwar Europe." World Politics 39, no. 4 (1987): 449-78.

Mackie, T. T., and R. Rose. The International Almanac of Electoral History. London: Macmillan (third edition), 1991.

Maddison, A. Statistics on World Population, GDP, and Per Capita GDP, 1-2008 AD. Groningen Growth and Development Center, University of Groningen, available at www.ggdc.net/MADDISON (2010).

McDonald, J. F., and R. A. Moffitt. "The Uses of Tobit Analysis.” Review of Economics and Statistics 62, no. 2 (1980): 318-21.

Moore, B. Jr. Social Origins of Dictatorship and Democracy: Lord and Peasant in the Making of the Modern World. Boston: Beacon Press, 1966.

Nohlen, D. Elections in the Americas: A Data Handbook, Vol. 2: South America. New York: Oxford University Press, 2005.

Nohlen, D., and P. Stöver. Elections in Europe: A Data Handbook. Baden-Baden: Nomos Verlagsgesellschaft, 2010.

O’Loughlin, J. "Can King's Ecological Inference Method Answer a Social Science Puzzle: Who Voted for the Nazi Party in the 1930s?" Annals of the Association of American Geographers 90, no. 3 (2000): 592-601. 
Payne, S. G. Fascism: Comparison and Definition. London and Madison: University of Wisconsin Press, 1983. . A History of Fascism: 1914-1945. London: Routledge, 1996.

Persson, T., and G. Tabellini. "Democratic Capital: The Nexus of Political and Economic Change." American Economic Journal: Macroeconomics 1, no. 2 (2009): 88-126.

Polity IV Database. Regime Authority Characteristics and Transitions, available at http://www.systemicpeace.org/inscr/inscr.htm (2009).

Ponticelli, J., and H.-J. Voth. "Austerity and Anarchy: Budget Cuts and Social Unrest in Europe, 1919-2009.” CEPR Discussion Paper No.8513, August 2011.

Putnam, R. D., R. Leonardi, and R. Y. Nanetti. Making Democracy Work: Civic Traditions in Modern Italy. Princeton, NJ: Princeton University Press, 1993.

Sartori, G. Parties and Party Systems. Cambridge: Cambridge University Press, 1976.

Sternberger, D., and B. Vogel. Die Wahl der Parlamente und anderer Staatsorgane, Band I: Europa, Erster Halbband. Berlin: Walter de Gruyter \& Co., 1969.

Stögbauer, C. "The Radicalisation of the German Electorate: Swinging to the Right and the Left in the Twilight of the Weimar Republic." European Review of Economic History 5, no. 2 (2001): 251-80.

Voigtlaender, N., and H.-J. Voth. "Persecution Perpetuated: The Medieval Origins of Anti-Semitic Violence in Nazi Germany." NBER Working Paper No. 17113, Cambridge, MA, June 2011.

Weitz, E. D. Creating German Communism, 1890-1990: From Popular Protests to Socialist State. Princeton, NJ: Princeton University Press, 1997.

Wooldridge, J. M. Econometric Analysis of Cross Section and Panel Data: Second Edition. Cambridge, MA: MIT Press, 2010. 